\title{
Characterizing Zircon Porphyry Copper Fertility Characteristics in the Evolving Laramide arc, east-central Arizona
}

Taylor J. Ledoux*, Craig J.R. Hart, and Robert G. Lee 


\section{Demand for Copper}

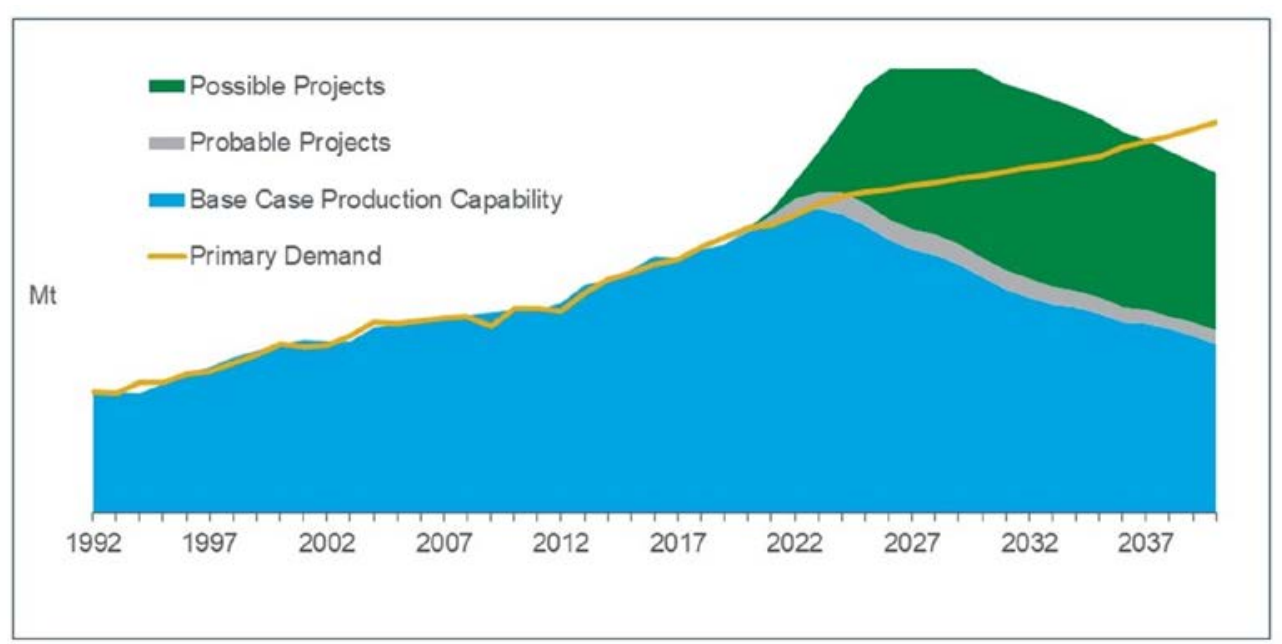

Wood Mackenzie (2019)
Copper usage has increased by $\mathbf{3 . 4 \%}$ annually since the 1900's.

Reliance on renewable energy and EV's will continue to drive global demand.

New discoveries are needed to meet Cu demand. 


\section{Cost and Value in Exploration: 1975-2018}

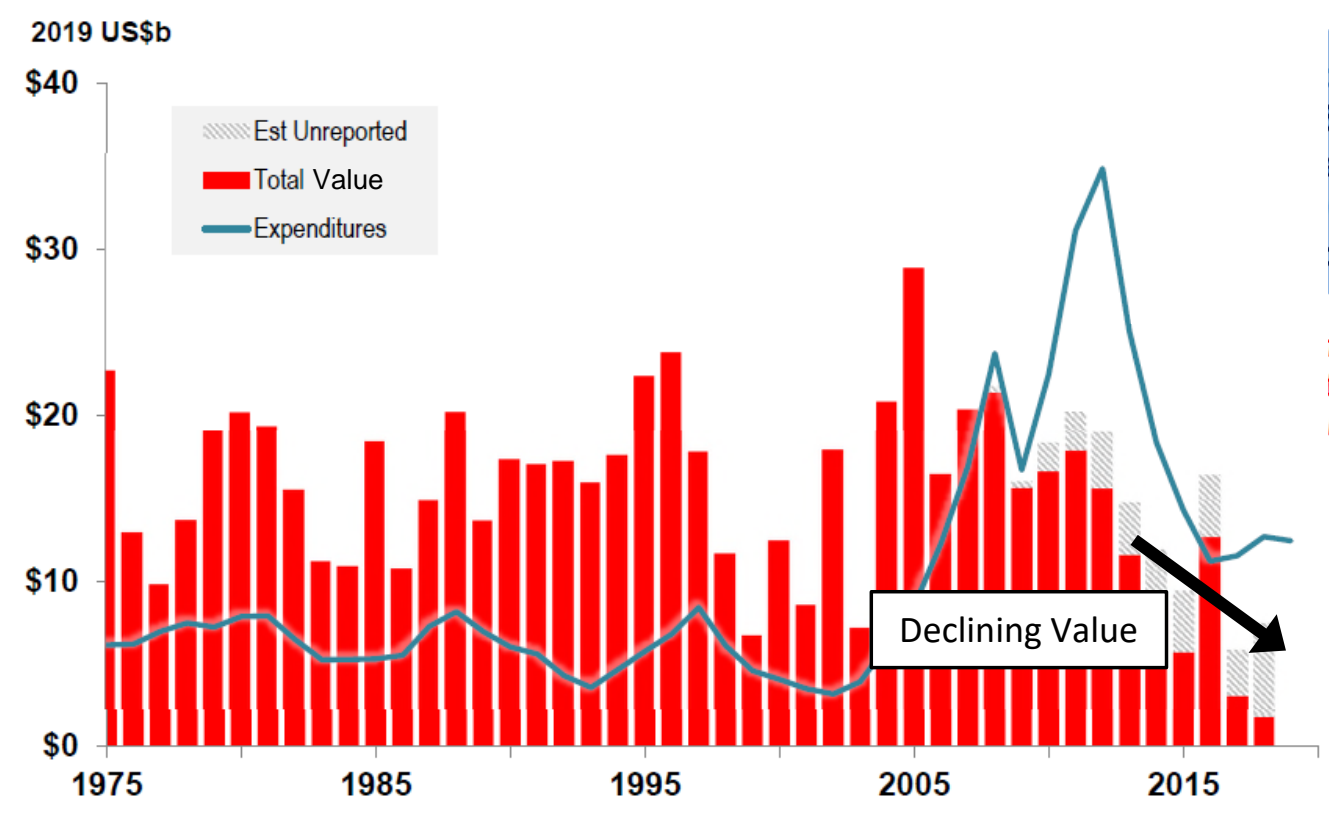

Schodde (2019)
Conventional Exploration Methods are ineffective

New Tools are needed to identify deposits and asses their economic potential 


\section{Porphyry Magma Fertility - Trace Elements in Zircon (TEZ)}

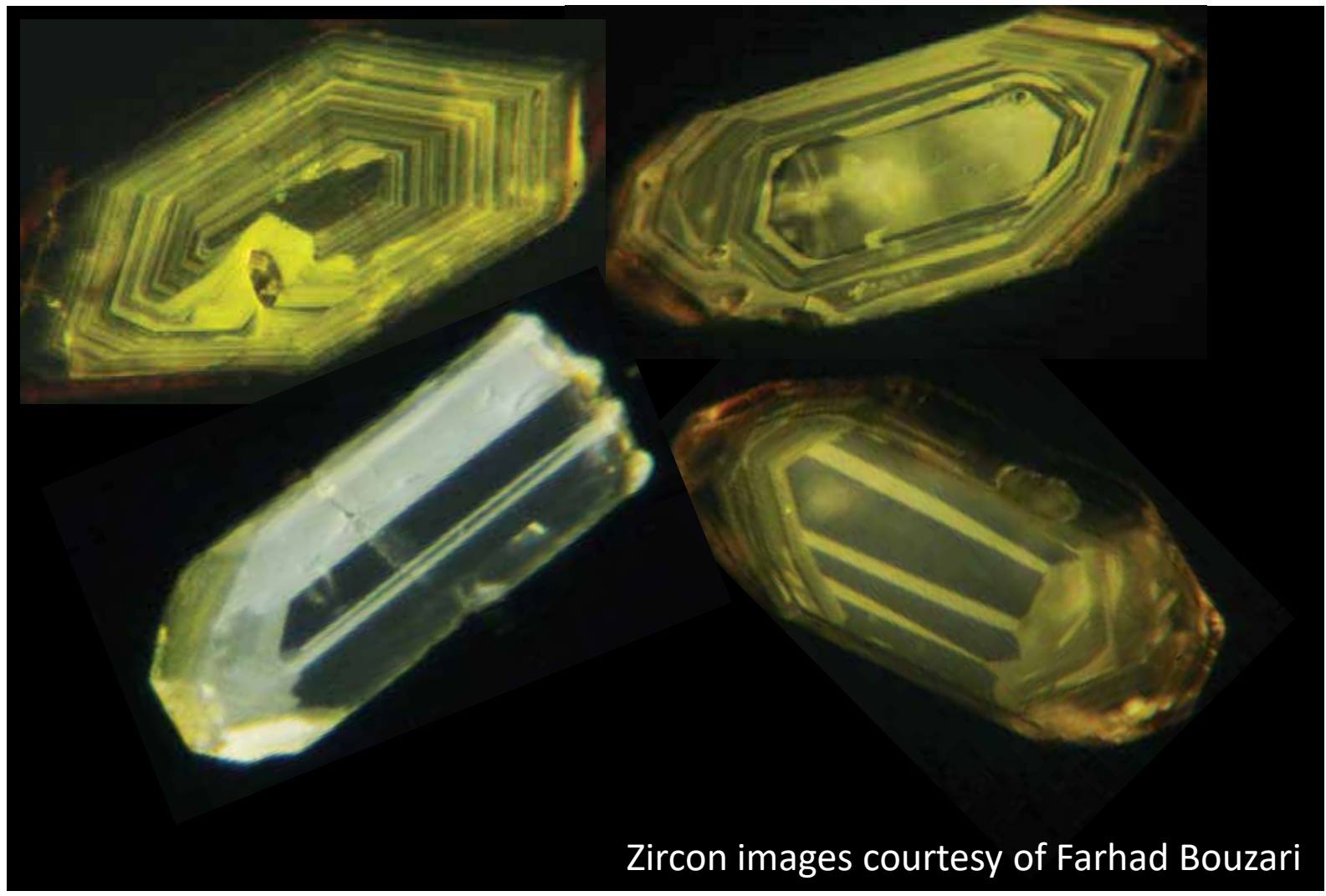




\section{Regional Geology}

\section{Variable Proterozoic Continental Basement}

\section{Laramide Arc}

- Arc magmatism migrated eastward

- Composite intrusive complexes

- Dioritic to granitic intrusions

- Magma Composition evolved to be more silicic

- Mineralizing intrusions during final stages of magmatism

Cenozoic Extension

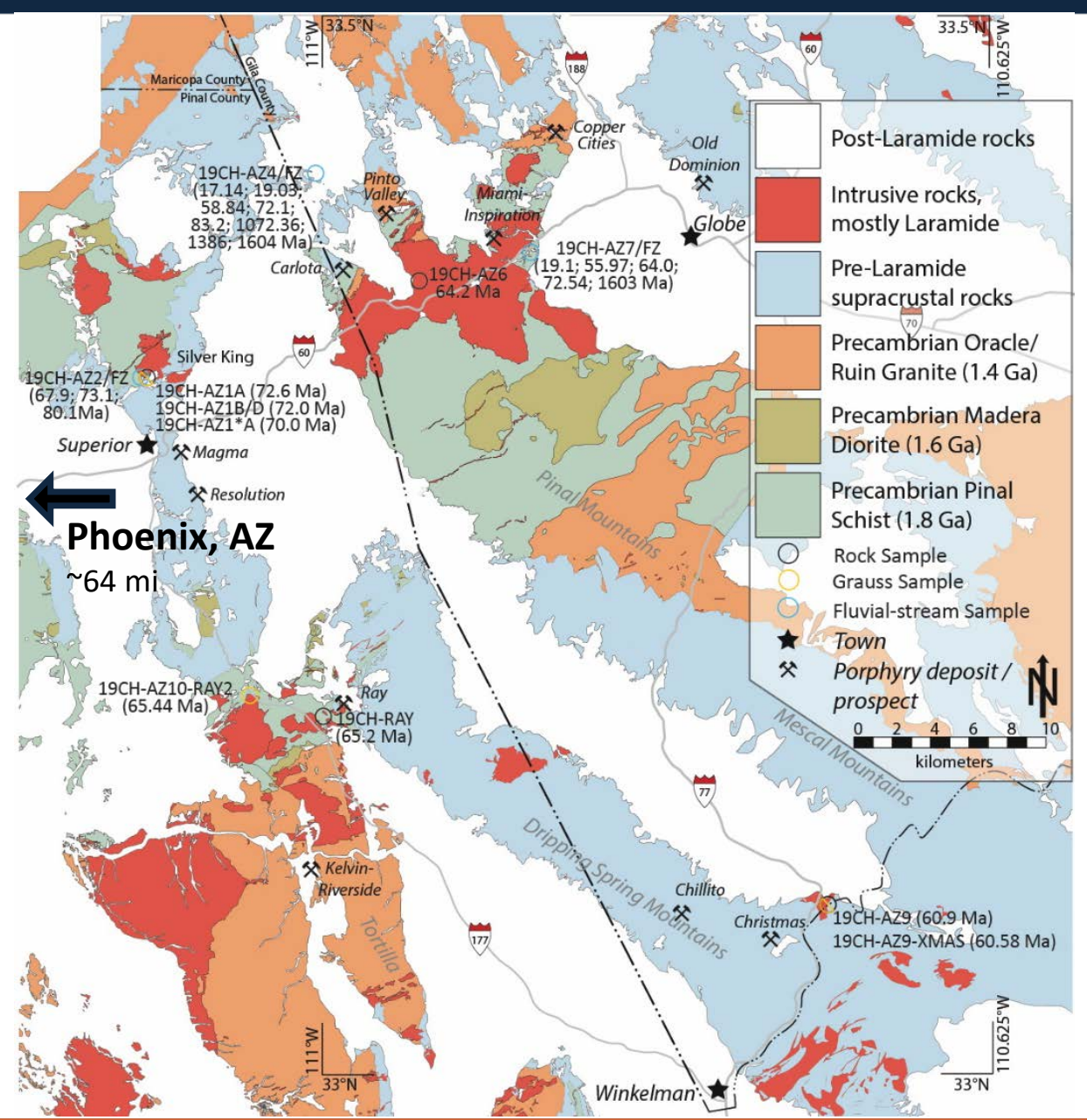




\section{Previous Geochronology}

\section{Superior District (ca. 75.2 to $63.5 \mathrm{Ma}$ )}

- Silver King ca. 75.2 to $72 \mathrm{Ma}$

- Magma Vein ca. 73.1 to $65.1 \mathrm{Ma}$

- Resolution ca. 70.4 to $63.5 \mathrm{Ma}$

- Superior East ca. 65 to $63.5 \mathrm{Ma}$

Globe-Miami District (ca. 69.8 to 59.9 Ma)

- Schultze Pluton (ca. 69.8 to 59.9 Ma)

- Pinto Valley/Carlota (ca. 64.1 to 63.1 Ma)

- Miami-Inspiration (ca.65.3 to 62.0 Ma)

Christmas (ca. 66.1 to 63.9 Ma)

Ray (ca. 72.1 to $64.4 \mathrm{Ma}$ )

(Hehnke et al., 2012; Seedorff et al., 2019)

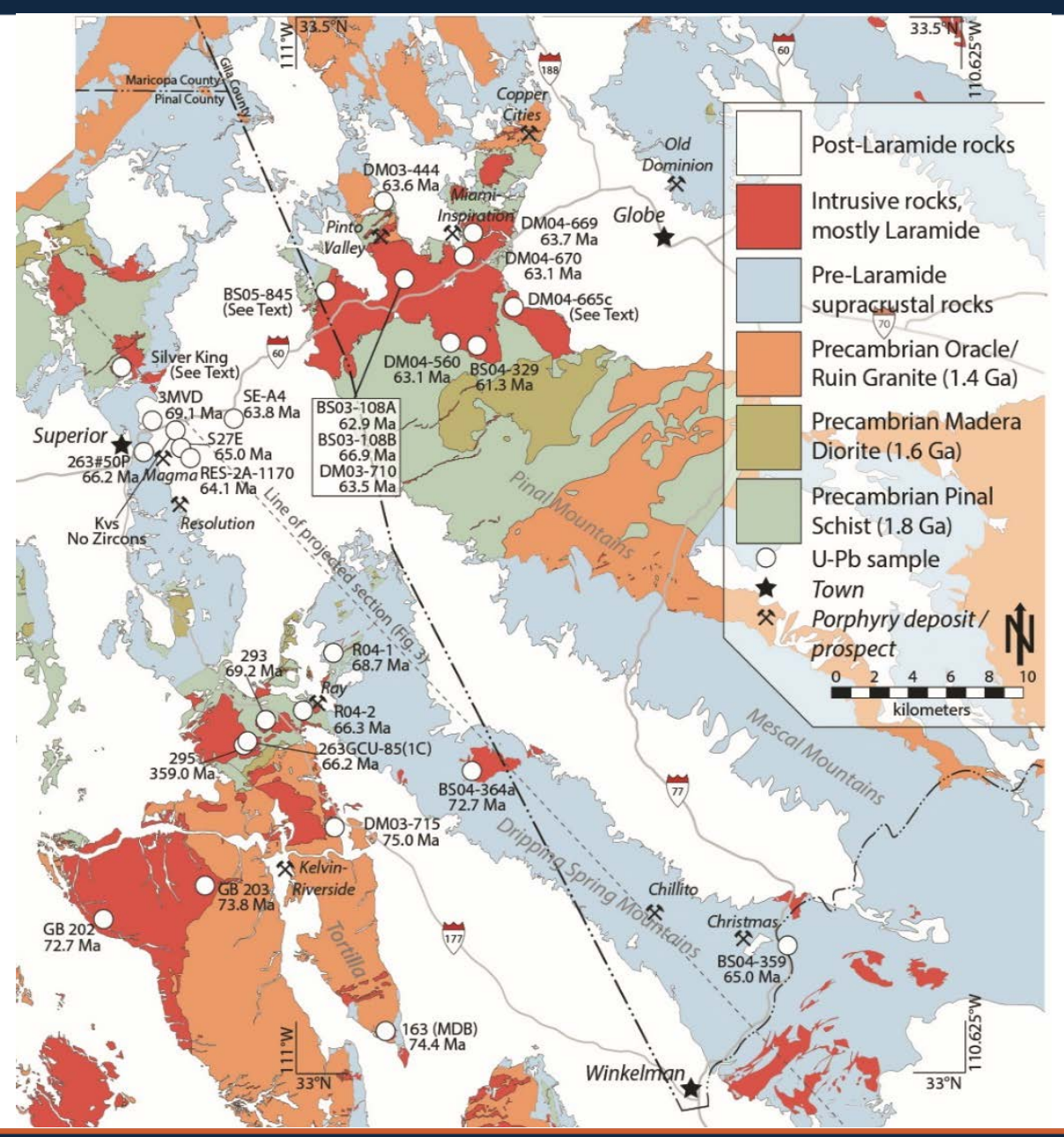




\section{Samples}

\section{Superior District - Silver King}

- $19 \mathrm{CH}-\mathrm{AZ1A}$ (equigranular quartz diorite stock)

- 19CH-AZ1B/D (equigranular monzonite dyke)

- 19CH-AZ1*A (grauss)

- 19CH-AZ2/FZ (fluvial-sand)

Globe-Miami District

- 19CH-AZ6 (porphyritic granodiorite)

- 19CH-AZ4/FZ (fluvial-sand)

- 19CH-AZ7/FZ (fluvial-sand)

\section{Christmas}

- $19 \mathrm{CH}-\mathrm{AZ9}$ (porphyritic monzodiorite)

- 19CH-AZ9-XMAS (grauss)

\section{Ray}

- 19CH-RAY (porphyritic granite)

- 19CH-AZ10-RAY2 (grauss)

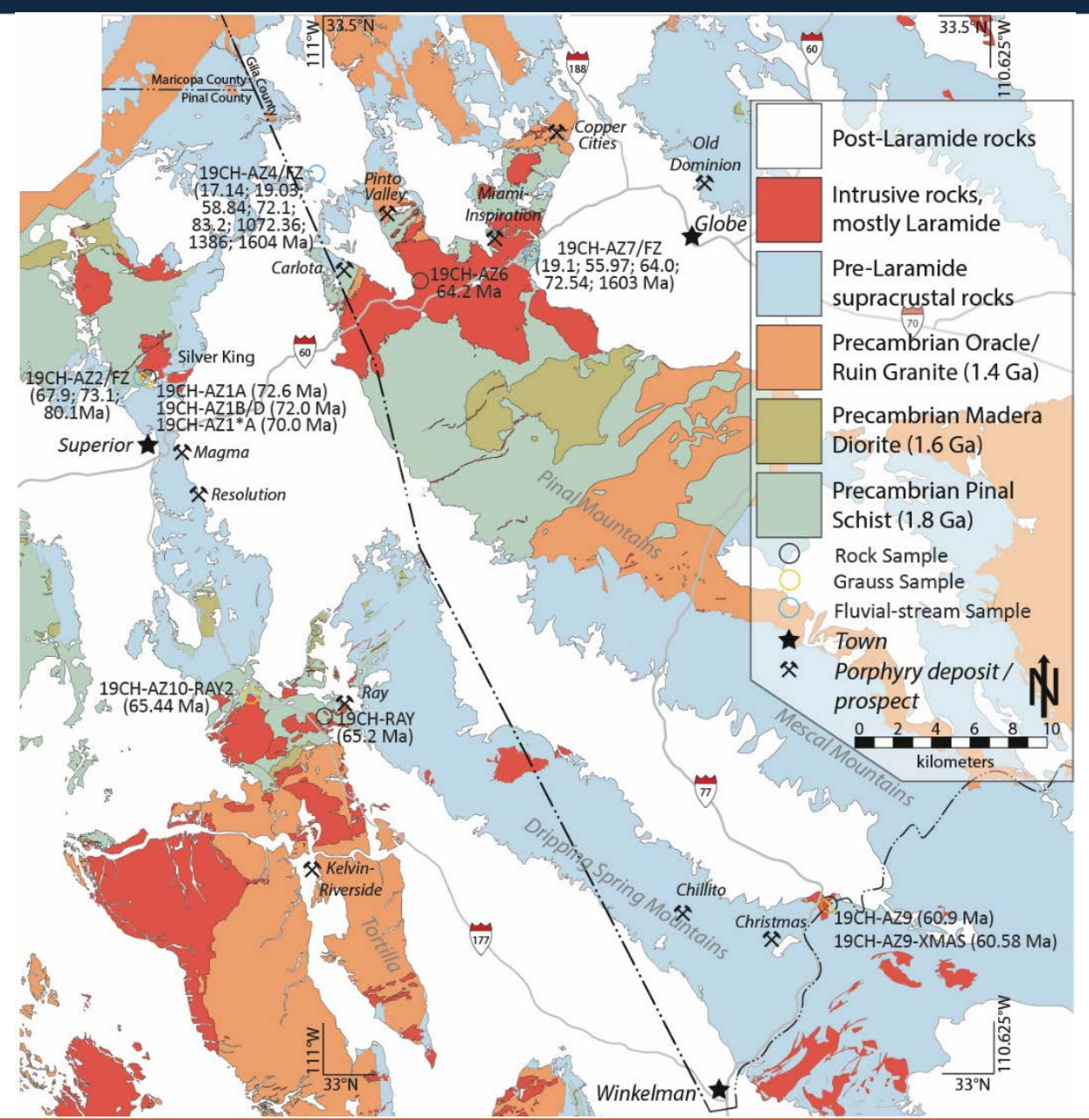




\section{Samples}

\section{Superior District - Silver King}

- $19 \mathrm{CH}-\mathrm{AZ1A}$ (equigranular quartz diorite stock)

- 19CH-AZ1B/D (equigranular monzonite dyke)

- 19CH-AZ1*A (grauss)

- 19CH-AZ2/FZ (fluvial-sand)

Globe-Miami District

- 19CH-AZ6 (porphyritic granodiorite)

- 19CH-AZ4/FZ (fluvial-sand)

- 19CH-AZ7/FZ (fluvial-sand)

\section{Christmas}

- $19 \mathrm{CH}-\mathrm{AZ9}$ (porphyritic monzodiorite)

- 19CH-AZ9-XMAS (grauss)

\section{Ray}

- 19CH-RAY (porphyritic granite)

- 19CH-AZ10-RAY2 (grauss)

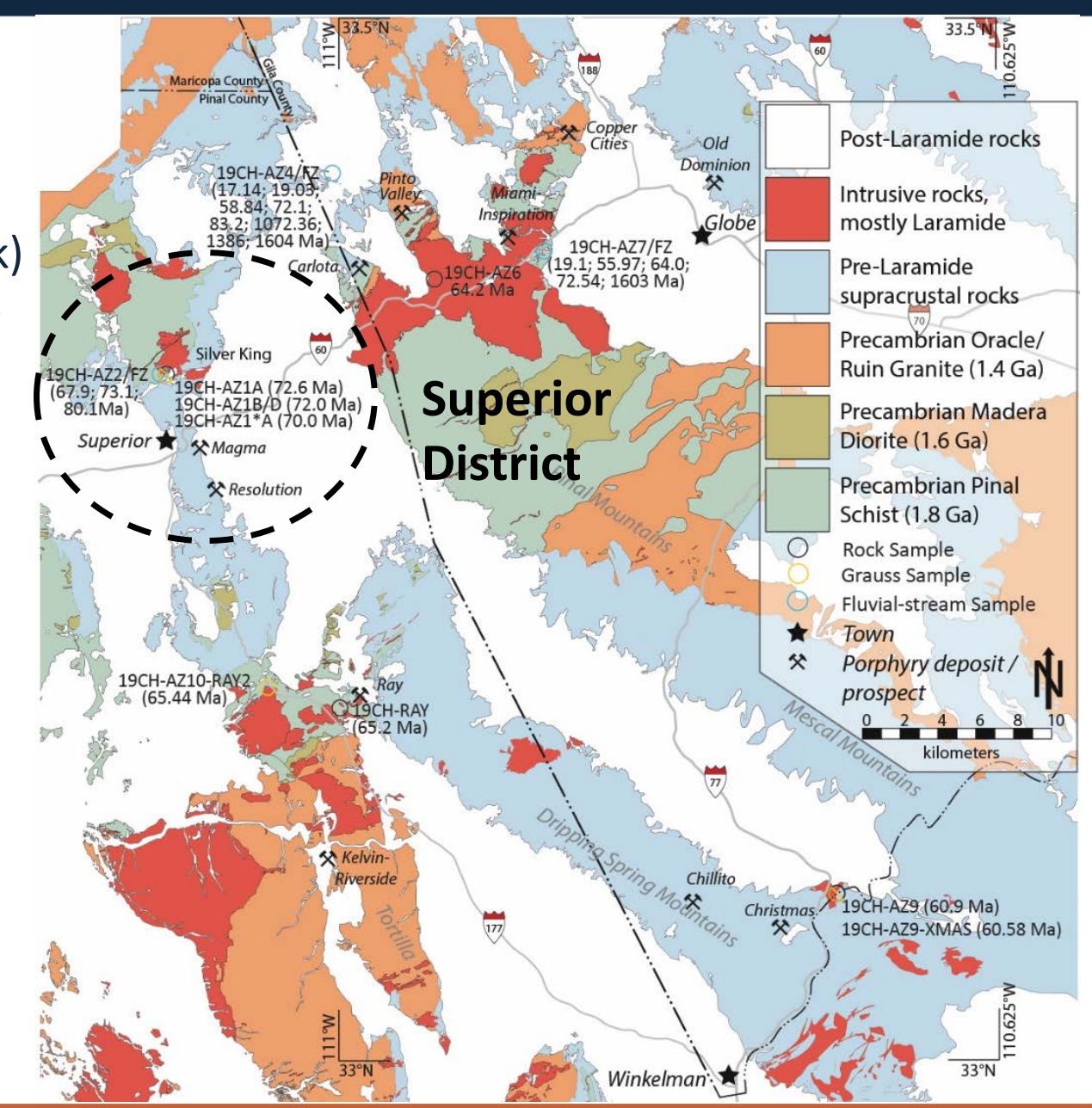




\section{Samples}

\section{Superior District - Silver King}

- $19 \mathrm{CH}-\mathrm{AZ1A}$ (equigranular quartz diorite stock)

- 19CH-AZ1B/D (equigranular monzonite dyke)

- 19CH-AZ1*A (grauss)

- 19CH-AZ2/FZ (fluvial-sand)

\section{Globe-Miami District}

- 19CH-AZ6 (porphyritic granodiorite)

- 19CH-AZ4/FZ (fluvial-sand)

- 19CH-AZ7/FZ (fluvial-sand)

\section{Christmas}

- $19 \mathrm{CH}-\mathrm{AZ9}$ (porphyritic monzodiorite)

- 19CH-AZ9-XMAS (grauss)

\section{Ray}

- 19CH-RAY (porphyritic granite)

- 19CH-AZ10-RAY2 (grauss)

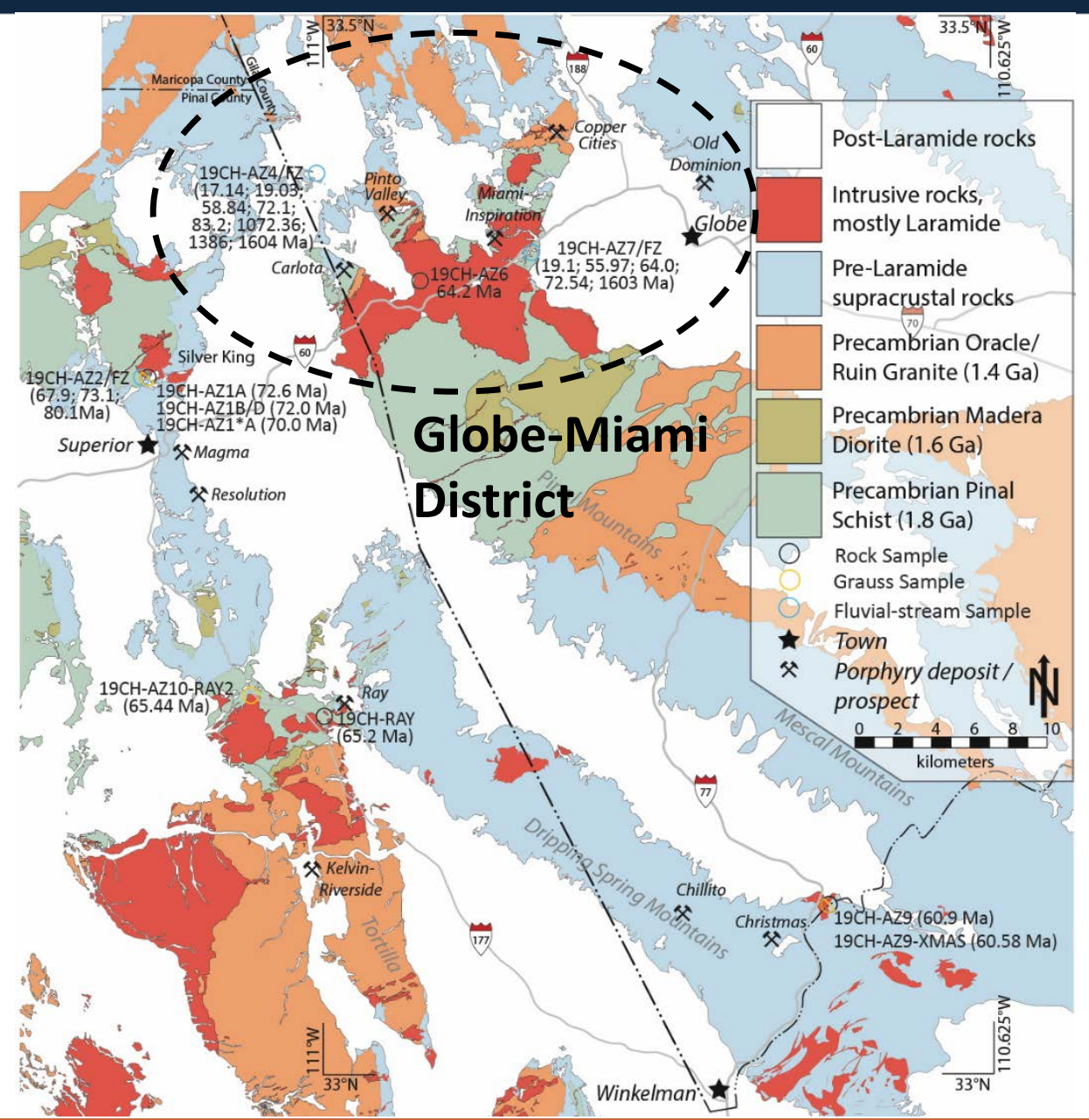




\section{Samples}

\section{Superior District - Silver King}

- $19 \mathrm{CH}-A Z 1 \mathrm{~A}$ (equigranular quartz diorite stock)

- 19CH-AZ1B/D (equigranular monzonite dyke)

- 19CH-AZ1*A (grauss)

- 19CH-AZ2/FZ (fluvial-sand)

\section{Globe-Miami District}

- 19CH-AZ6 (porphyritic granodiorite)

- 19CH-AZ4/FZ (fluvial-sand)

- 19CH-AZ7/FZ (fluvial-sand)

\section{Christmas}

- 19CH-AZ9 (porphyritic monzodiorite)

- 19CH-AZ9-XMAS (grauss)

\section{Ray}

- 19CH-RAY (porphyritic granite)

- 19CH-AZ10-RAY2 (grauss)

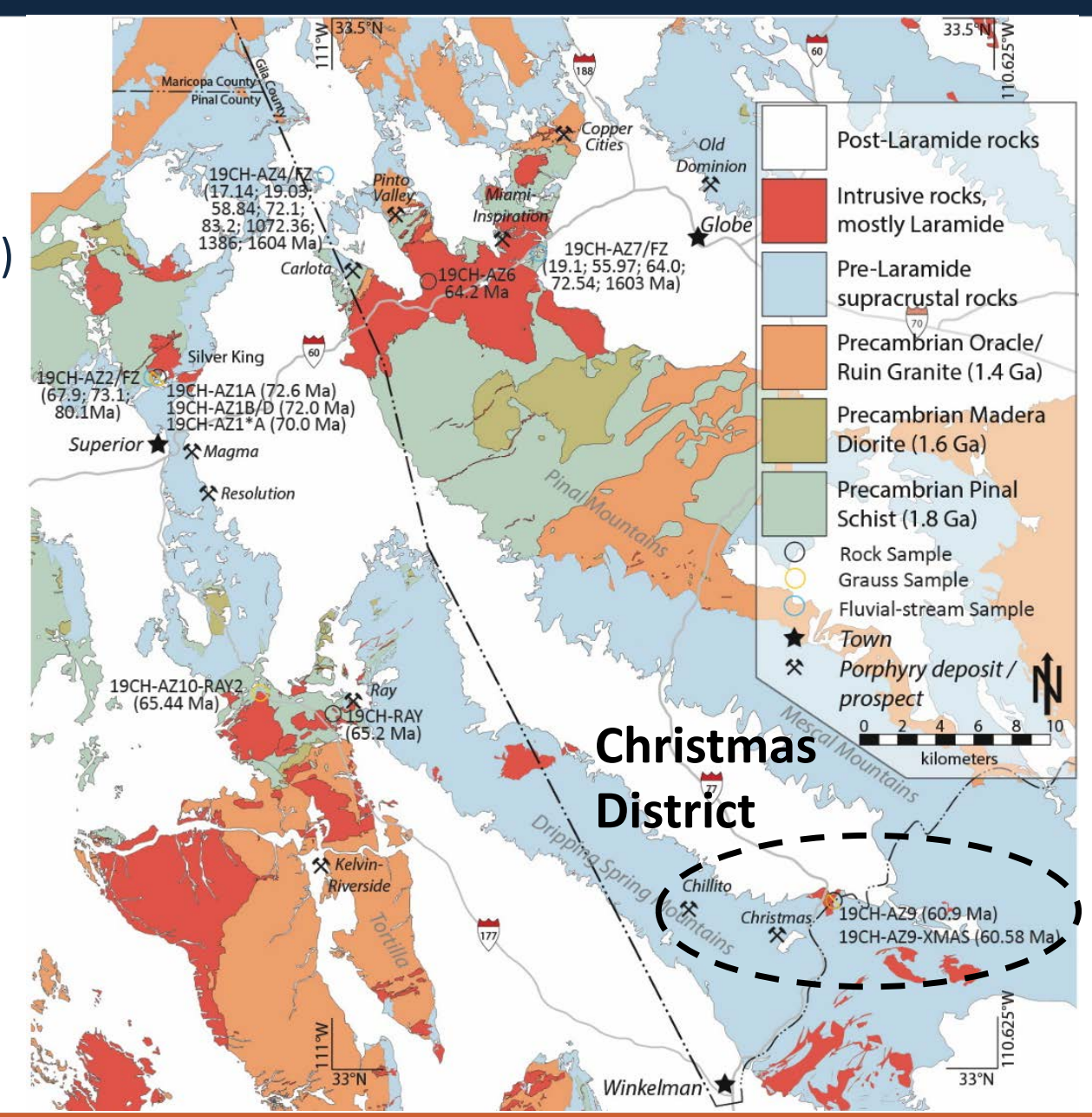




\section{Samples}

\section{Superior District - Silver King}

- $19 \mathrm{CH}-\mathrm{AZ1A}$ (equigranular quartz diorite stock)

- 19CH-AZ1B/D (equigranular monzonite dyke)

- 19CH-AZ1*A (grauss)

- 19CH-AZ2/FZ (fluvial-sand)

\section{Globe-Miami District}

- 19CH-AZ6 (porphyritic granodiorite)

- 19CH-AZ4/FZ (fluvial-sand)

- 19CH-AZ7/FZ (fluvial-sand)

\section{Christmas}

- $19 \mathrm{CH}-\mathrm{AZ9}$ (porphyritic monzodiorite)

- 19CH-AZ9-XMAS (grauss)

\section{Ray}

- 19CH-RAY (porphyritic granite)

- 19CH-AZ10-RAY2 (grauss)

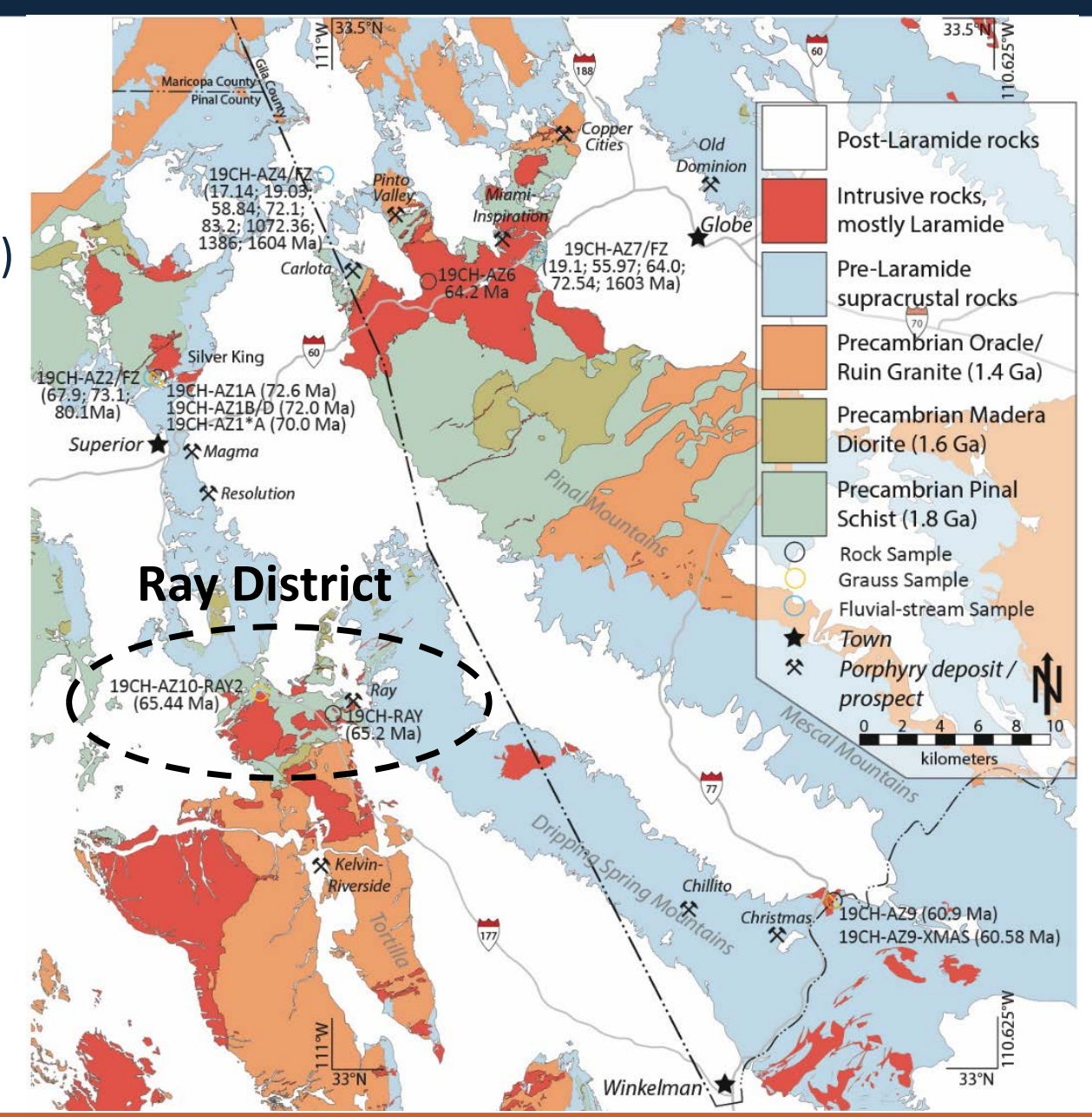




\section{Geochronology - Superior District}

\section{Silver King stock}

Quartz diorite stock 72.6 $\pm 2.9 \mathrm{Ma}$ - $72.6 \pm 2.9 \mathrm{Ma}$ autocrysts (25/25) Monzonite dike 72.0 \pm 2.3 Ma

- $\quad 72.0 \pm 2.3 \mathrm{Ma}$ autocrysts (4/15)

- $\quad 77.3 \pm 2.3 \mathrm{Ma}$ antecrysts (5/15)

- $1407 \pm 290$ Ma xenocrysts (6/15)

Silver King Grauss 70.3 $\pm 1.8 \mathrm{Ma}$

- $\quad 65.72 \pm 5.49 \mathrm{Ma}(1 / 9)$

- $70.3 \pm 1.8 \mathrm{Ma}(7 / 9)$

- $\quad 77.77 \pm 4.47 \mathrm{Ma}(1 / 9)$

Fluvial Sand 81.42 to $65.84 \mathrm{Ma}$

- Laramide 81.42 to $65.84 \mathrm{Ma}(57 / 57)$
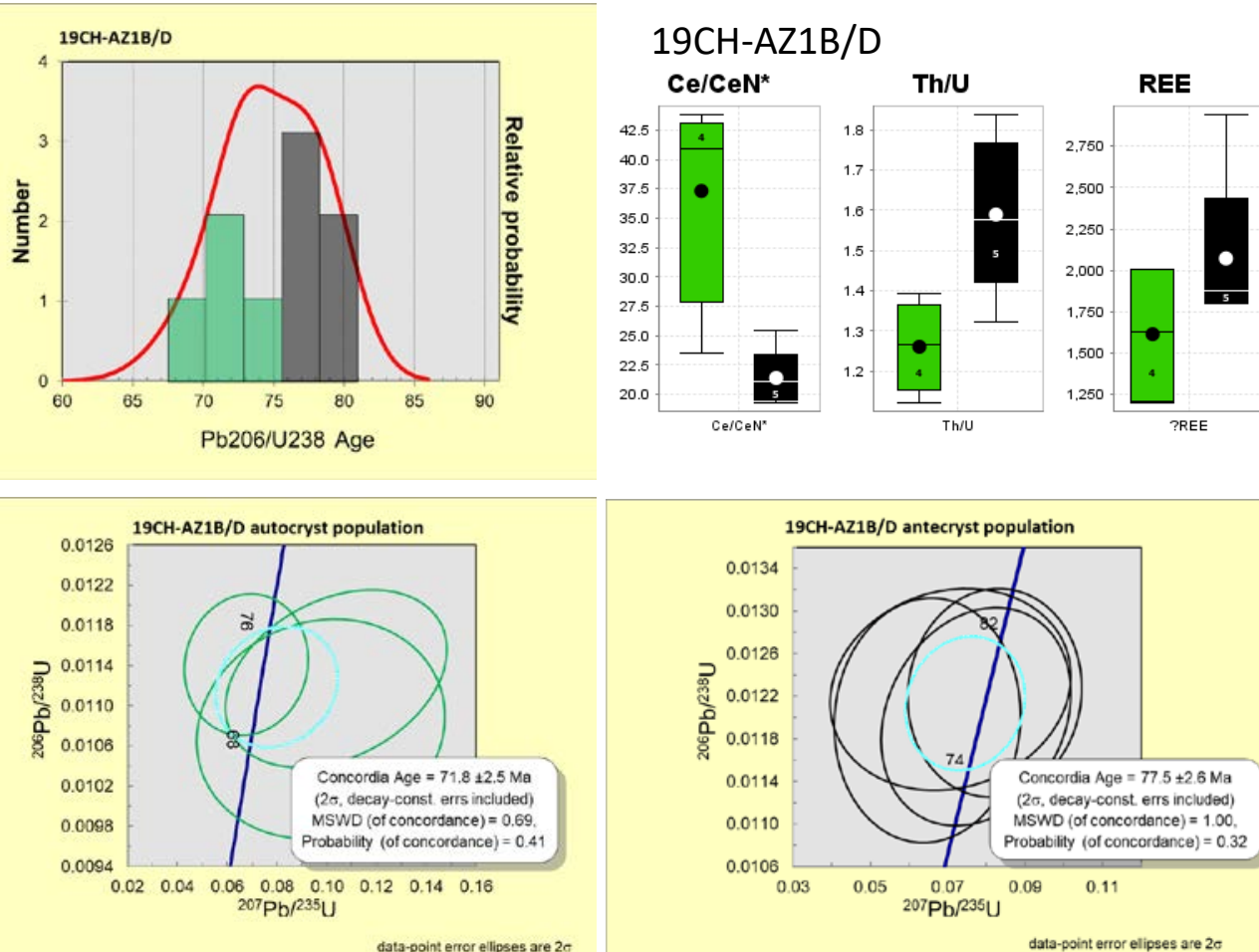


\section{Geochronology - Globe-Miami District (Schultze pluton)}

\section{Schultze pluton}

Porphyritic granodiorite 64.2ะ 3.1 Ma

- $\quad 64.2 \pm 3.1 \mathrm{Ma}$ autocrysts $(2 / 16)$

- $1579 \pm 90$ Ma xenocrysts (14/16)

\section{NW Pinto Valley}

Fluvial sand 16.34 to $1604 \mathrm{Ma}$

- Early Miocene 20.30 to 16.34 (34/53)

- Laramide 81.0 to $58.84 \mathrm{Ma}(10 / 53)$

- Proterozoic 1072, 1386, and $1604 \mathrm{Ma}$ (9/53)

SE Miami-Inspiration

Fluvial sand 18.42 to $1603 \mathrm{Ma}$

- Early Miocene 21.84 to $18.42(2 / 56)$

- Laramide 78.20 to $55.97(25 / 56)$

- Proterozoic $1603 \pm 69 \mathrm{Ma}(9 / 56)$
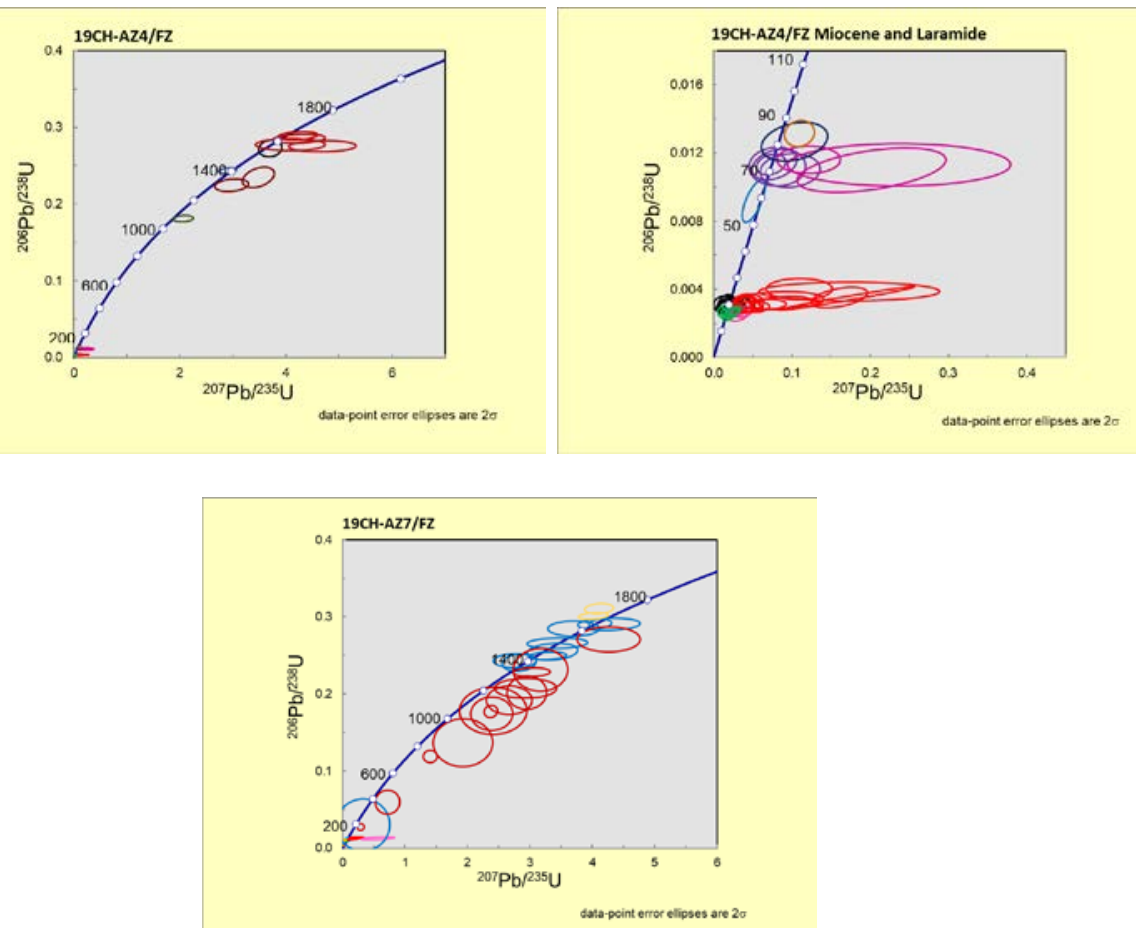


\section{Geochronology-Christmas}

\section{Christmas stock}

Porphyritic monzodiorite 60.9 1.3 Ma

- $\quad 60.9 \pm 1.3 \mathrm{Ma}$ autocrysts (11/30)

- $\quad 65.7 \pm 1.2 \mathrm{Ma}$ antecrysts (16/30)

- $1367 \pm 52$ Ma xenocrysts (3/30)

\section{Grauss $60.58 \pm 0.94 \mathrm{Ma}$}

- $\quad 60.58 \pm 0.94 \mathrm{Ma}$ autocrysts (14/28)

- $\quad 65.1 \pm 1.3 \mathrm{Ma}$ antecrysts (10/28)

- $1427 \pm 210$ Ma xenocrysts (4/28)

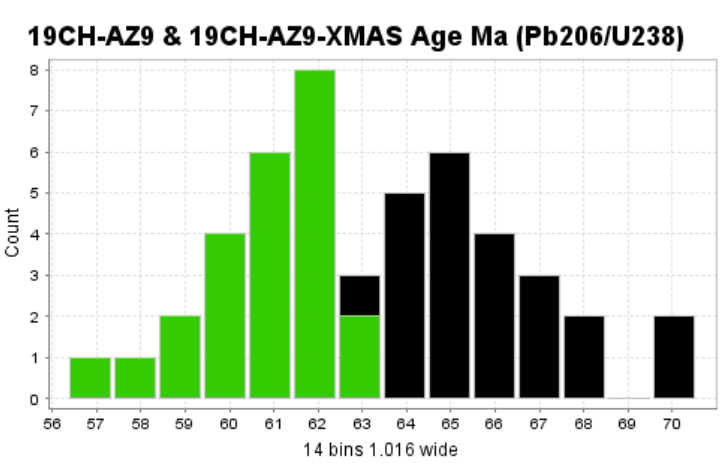

19CH-AZ9 \& 19CH-AZ9-XMAS
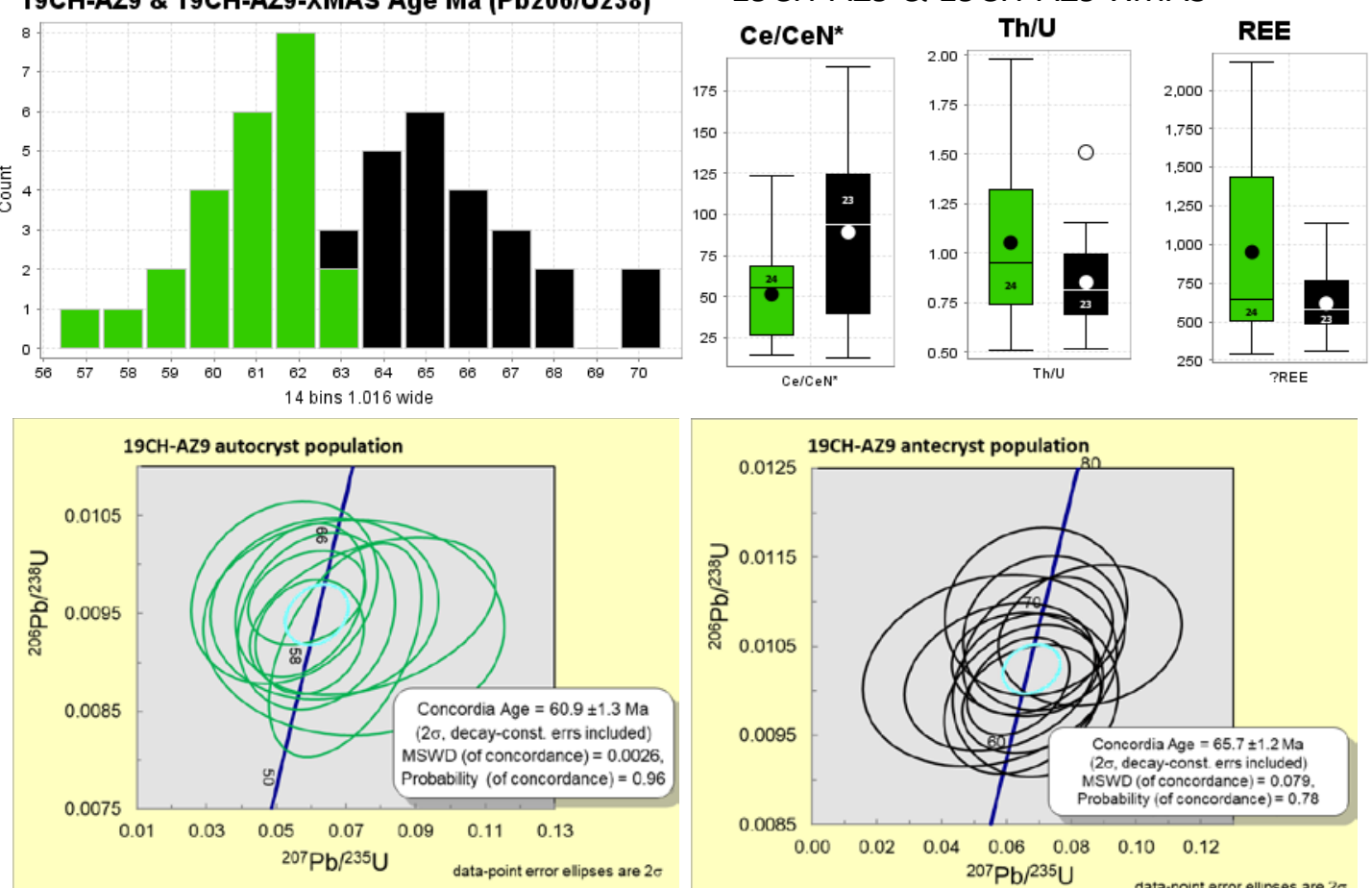


\section{Geochronology - Ray}

\section{Granite Mountain pluton}

Porphyritic granite $64.34 \pm 4.57 \mathrm{Ma}$

- 1 moderately discordant grain

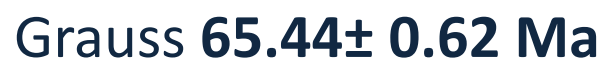

- $65.44 \pm 0.62 \mathrm{Ma}$ autocrysts (30/36)

- $\quad 1586 \pm 26$ Ma xenocrysts (6/36)
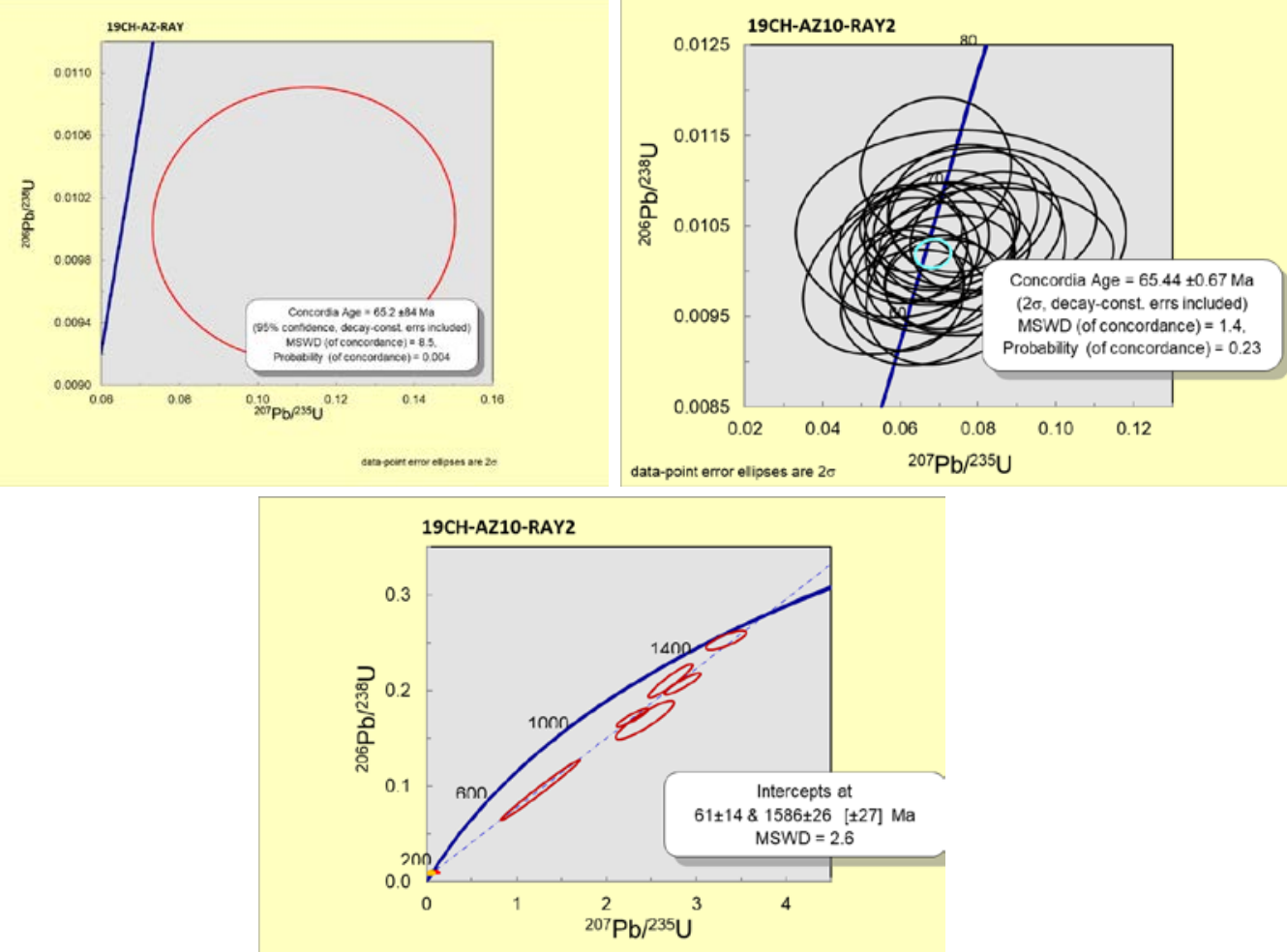

data-point error ellipses are $2 \sigma$ 


\section{Porphyry Magma Fertility}

Key Magmatic Parameters (Proxies in Zircon)

1) Oxidation State (Eu/Eu* $\left., \mathrm{Ce} / \mathrm{Ce}^{*}, \& \Delta \mathrm{FMQ}\right)$

2) Temperature (Ti-in-zircon-thermometer)

3) Water Content (Eu/Eu*)

4) Metal Content

5) Chlorine Content

6) Sulphur Content

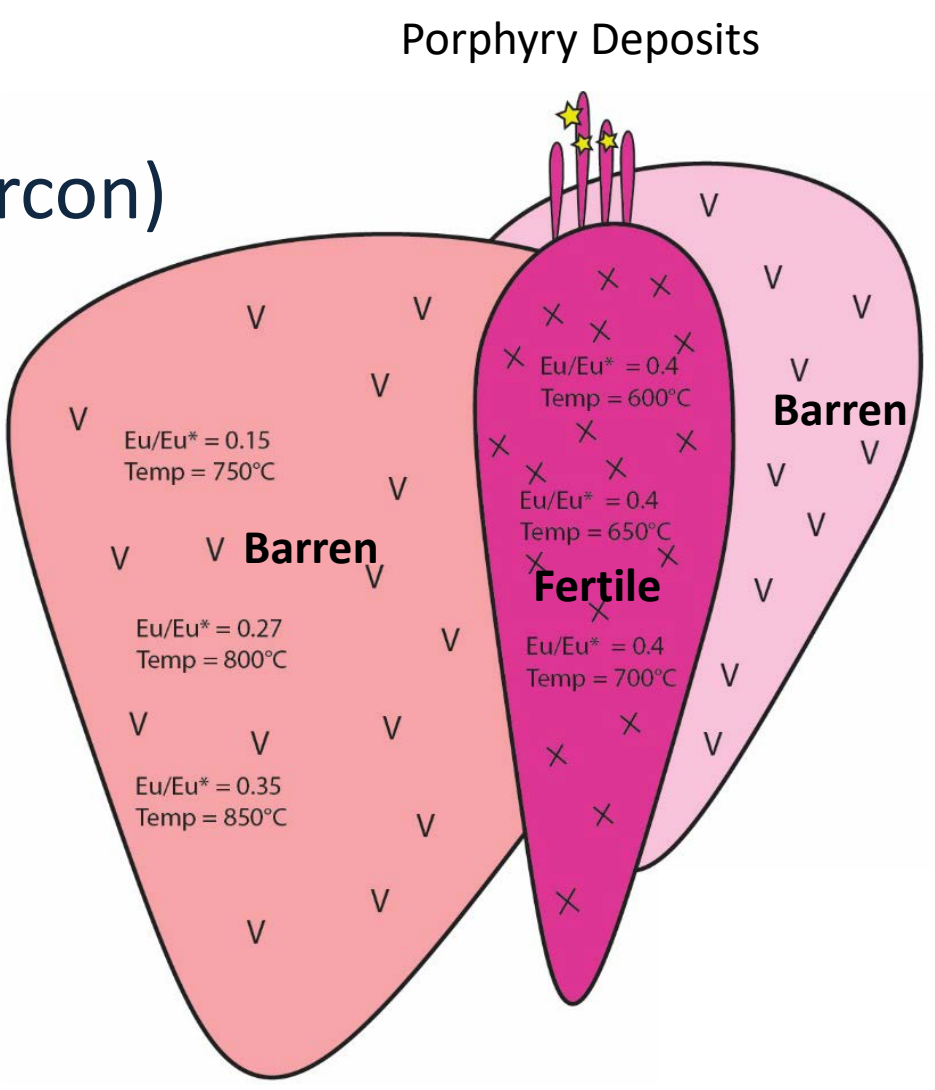




\section{Trace Eements in Zircon (TEZ)}

\section{Fractionation}

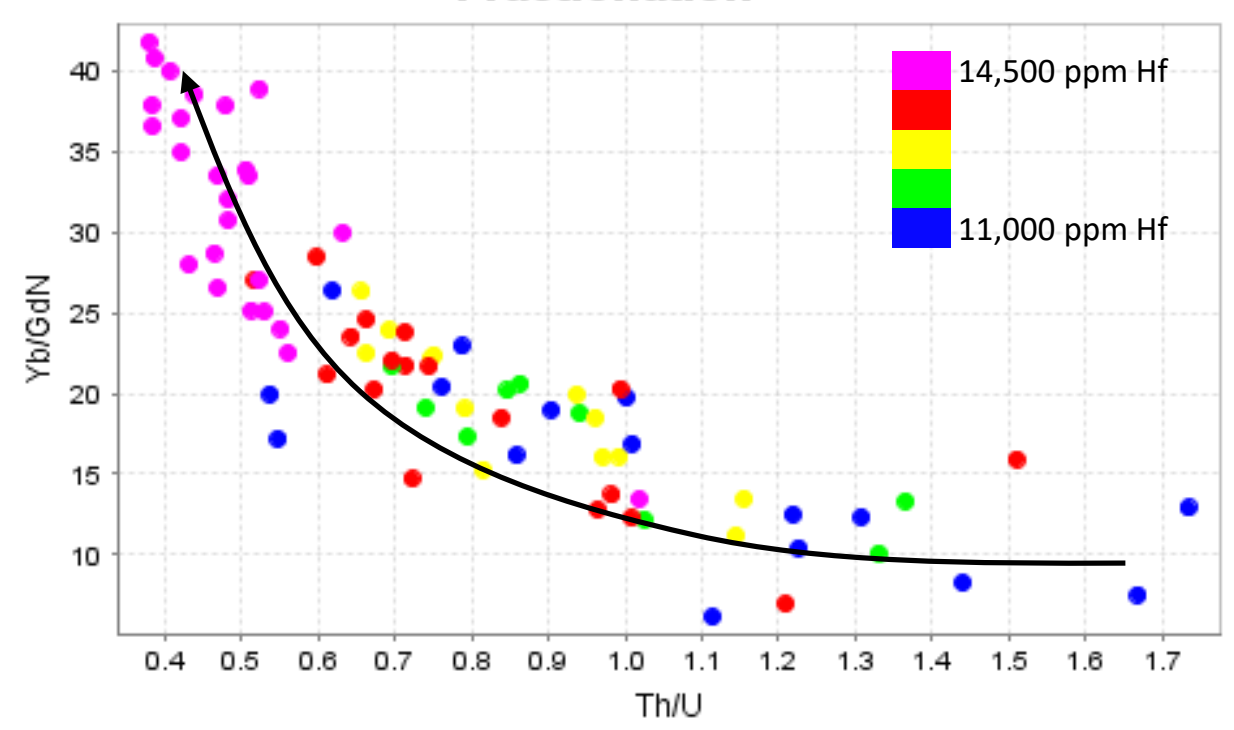

\section{Crystal Fractionation}

$\downarrow \mathrm{Th} / \mathrm{U}$

$\uparrow \mathrm{Yb} / \mathrm{Gd}_{\mathrm{N}}$

个 Hf 


\section{Trace Eements in Zircon (TEZ)}

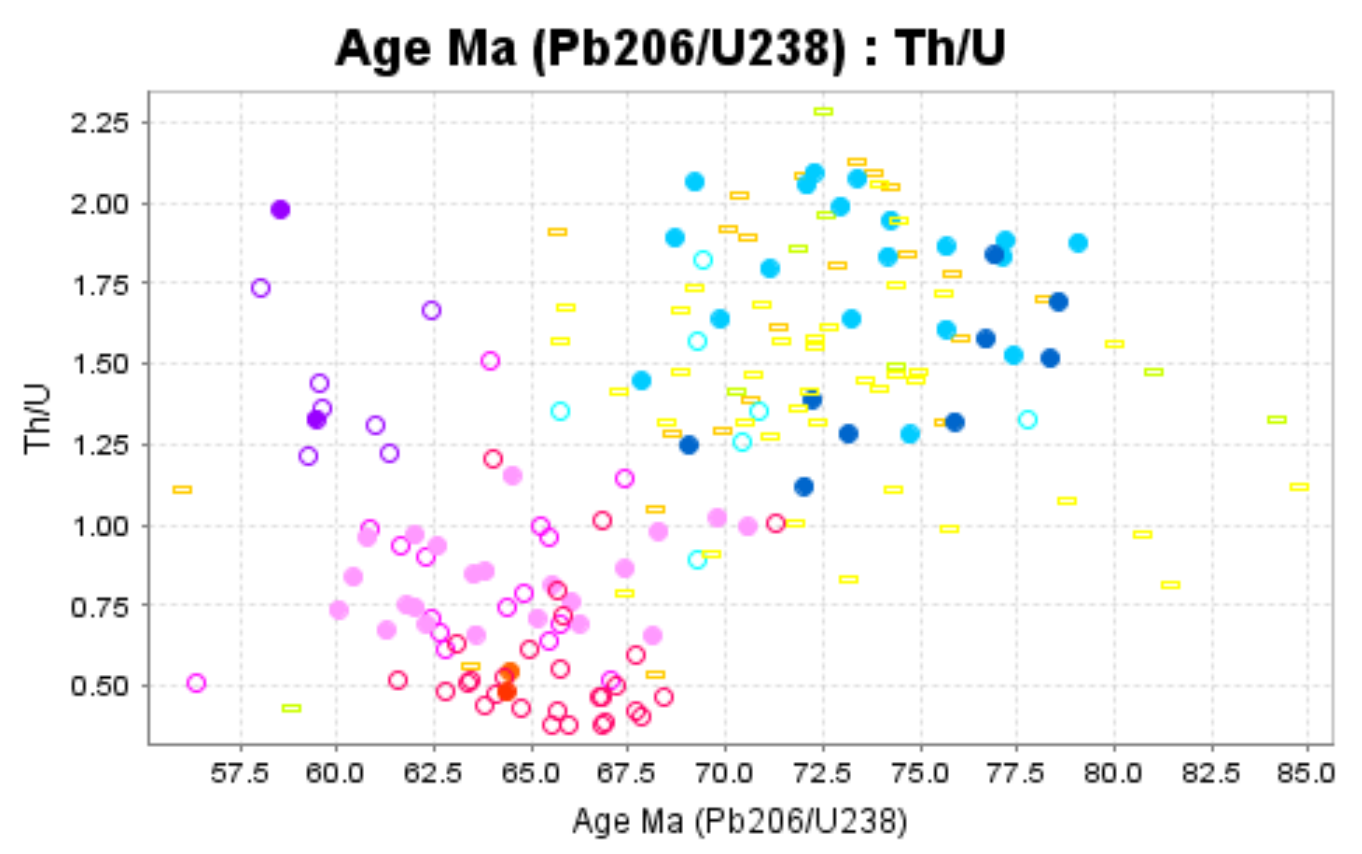

\section{Christmas}

$\bigcirc$ Monzodiorite (Grauss)

- Monzodiorite

- Monzodiorite, primative autocrysts

O Monzodiorite (Grauss), primative autocrysts Ray

O Granite (Grauss)

- Granite

\section{Globe-Miami}

- Granodiorite

- Fluvial-sand, Pinto Valley

- Fluvial-sand, Miami-Inspiration

\section{Silver King}

Fluvial-sand, Silver King

- Quartz diorite (Grauss)

- Quartz diorite

- Monzonite 


\section{Trace Eements in Zircon (TEZ)}

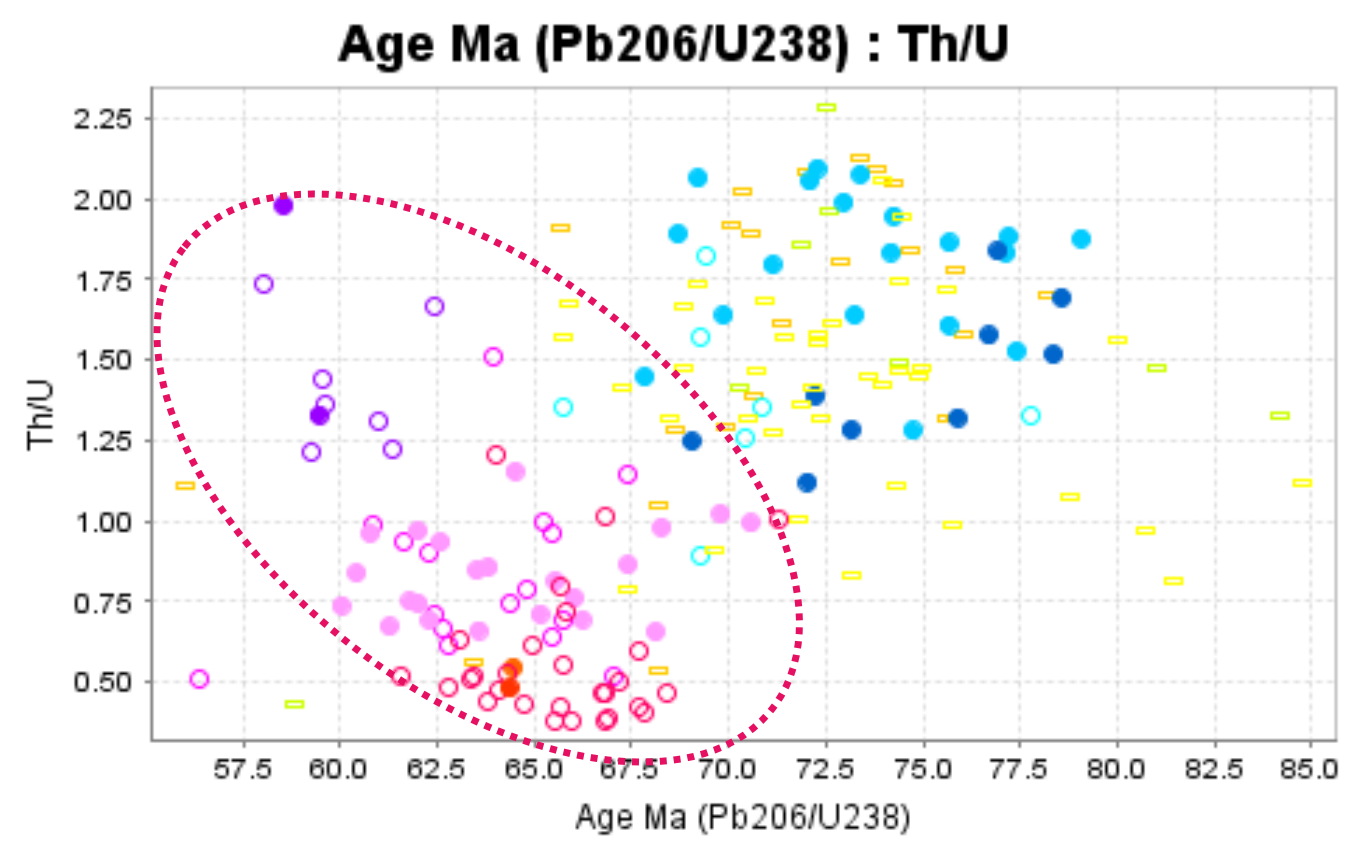

\section{Fertile young population}

\section{Christmas}

O Monzodiorite (Grauss)

- Monzodiorite

- Monzodiorite, primative autocrysts

O Monzodiorite (Grauss), primative autocrysts Ray

- Granite (Grauss)

- Granite

Globe-Miami

Granodiorite

- Fluvial-sand, Pinto Valley

- Fluvial-sand, Miami-Inspiration

\section{Silver King}

Fluvial-sand, Silver King

- Quartz diorite (Grauss)

- Quartz diorite

- Monzonite 


\section{Trace Eements in Zircon (TEZ)}

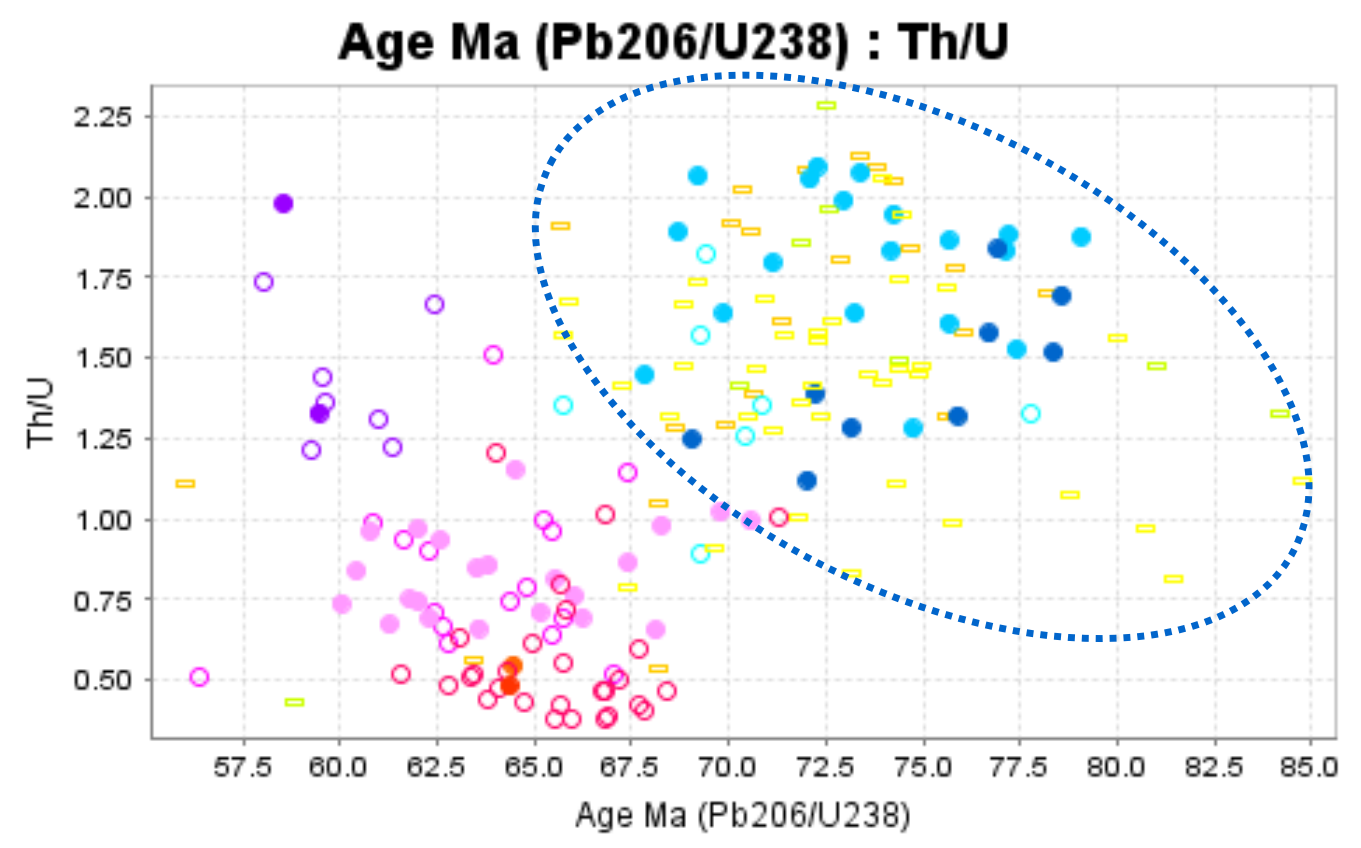

\section{Christmas}

$\bigcirc$ Monzodiorite (Grauss)

- Monzodiorite

- Monzodiorite, primative autocrysts

O Monzodiorite (Grauss), primative autocrysts Ray

O Granite (Grauss)

- Granite

Globe-Miami

Granodiorite Baren old population Fluvial-sand, Pinto Valley

- Fluvial-sand, Miami-Inspiration

\section{Silver King}

Fluvial-sand, Silver King

- Quartz diorite (Grauss)

- Quartz diorite

Monzonite 


\section{Trace Eements in Zircon (TEZ)}

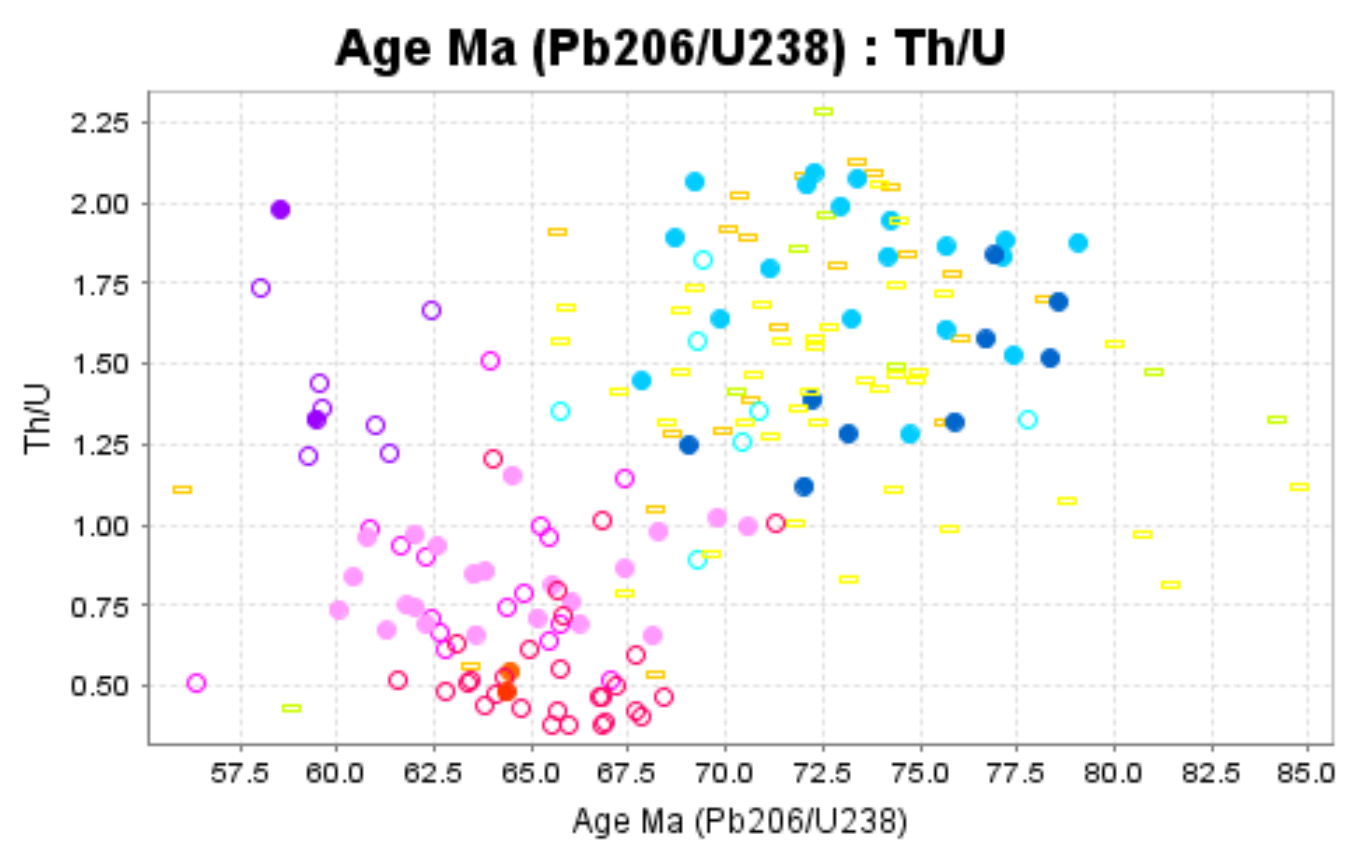

\section{Christmas}

$\bigcirc$ Monzodiorite (Grauss)

- Monzodiorite

- Monzodiorite, primative autocrysts

O Monzodiorite (Grauss), primative autocrysts Ray

O Granite (Grauss)

- Granite

\section{Globe-Miami}

- Granodiorite

- Fluvial-sand, Pinto Valley

- Fluvial-sand, Miami-Inspiration

\section{Silver King}

Fluvial-sand, Silver King

- Quartz diorite (Grauss)

- Quartz diorite

- Monzonite 


\section{Trace Eements in Zircon (TEZ) - Fractionation}

Th/U : Yb/GdN

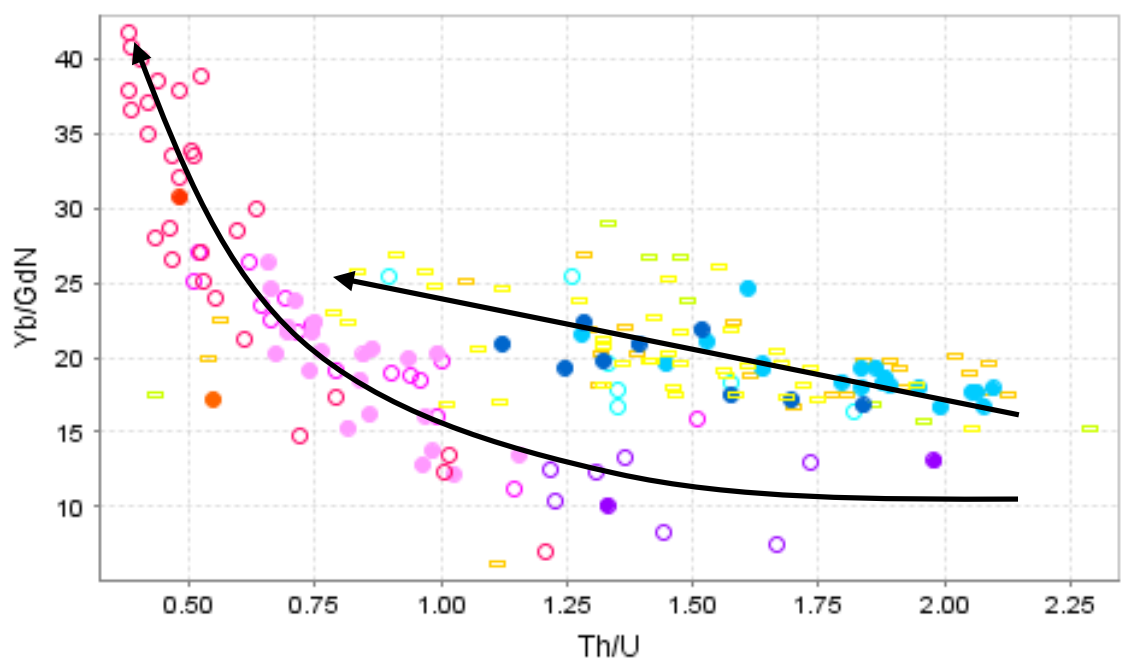

Th/U : Hf/Y

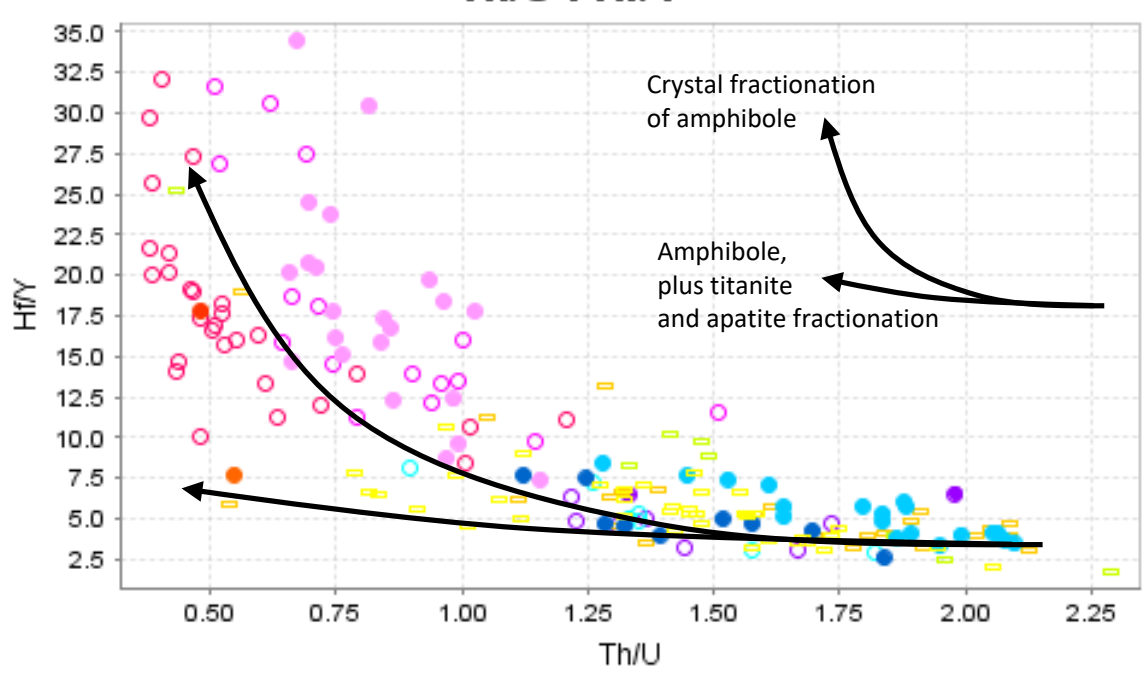

Fractionation trends after Lee et al., 2020 


\section{Trace Eements in Zircon (TEZ)}

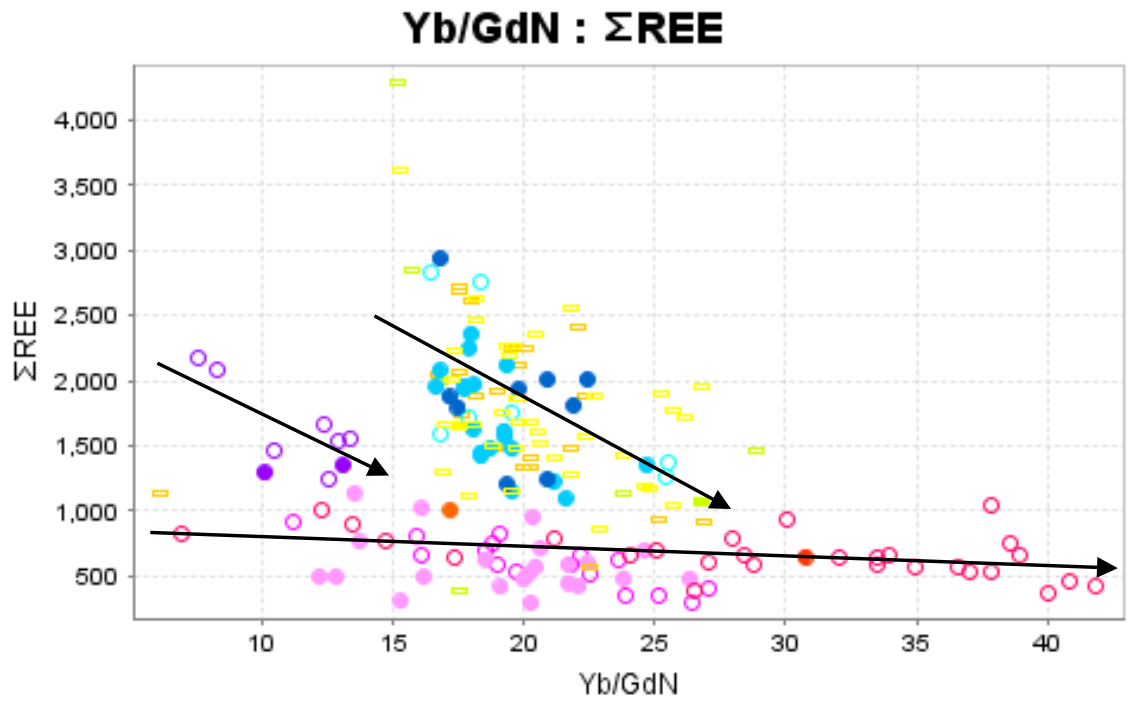

Hf_ppm : Temp $\mathrm{C}^{\circ}$ aTi=0.7

rtz diorite (Grauss)

Monzonite

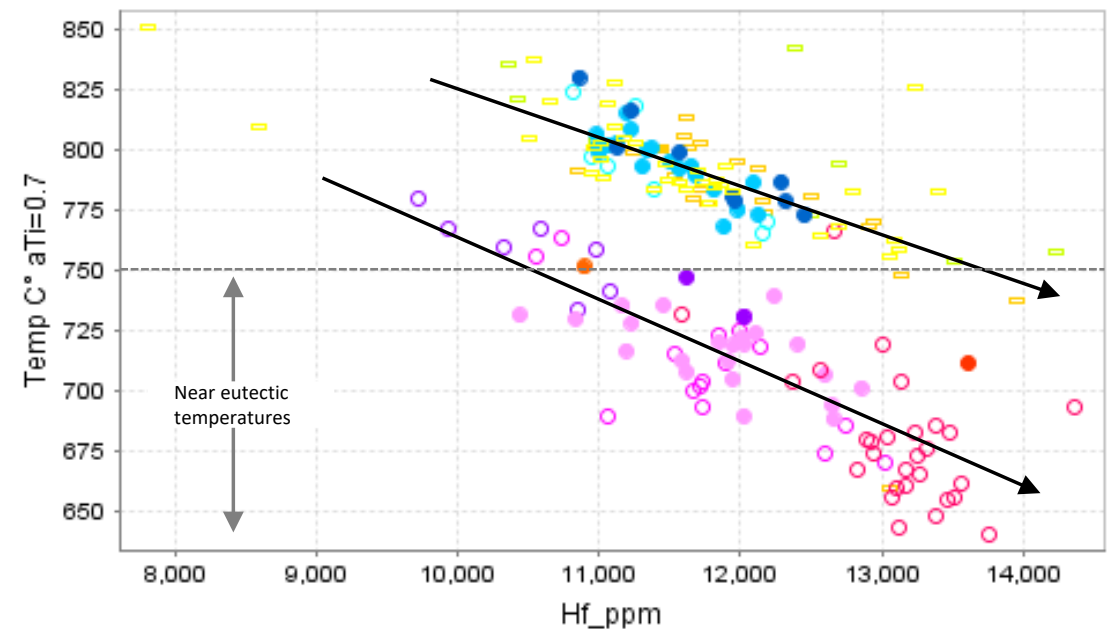




\section{Trace Eements in Zircon (TEZ) - Magma Recharge}
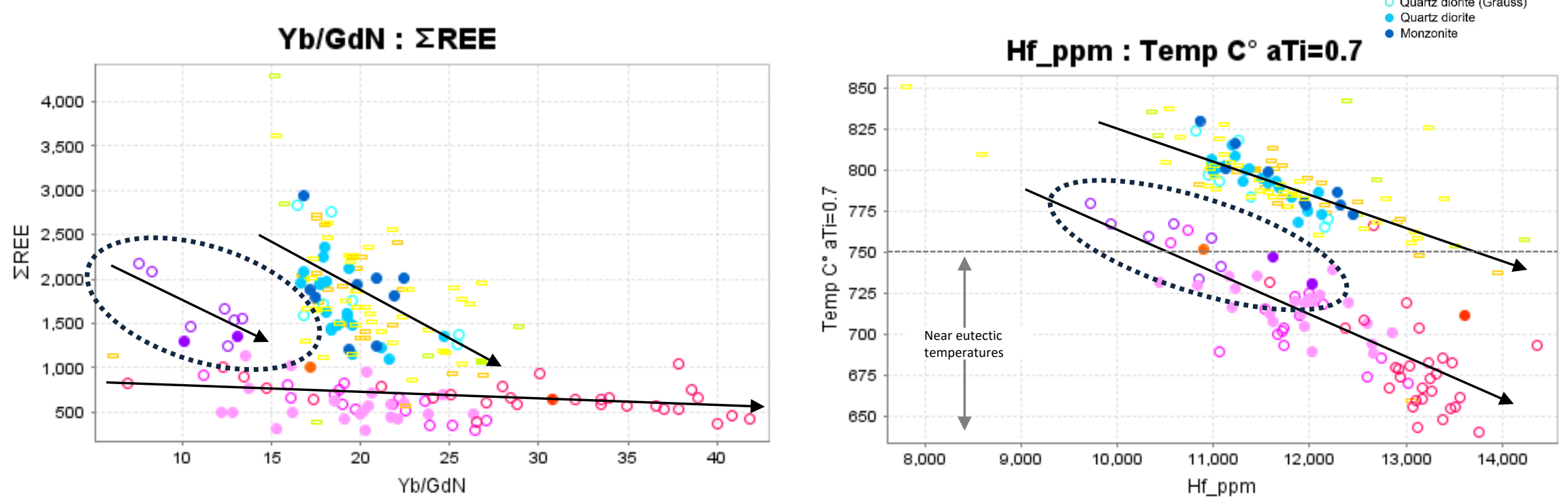


\section{Trace Eements in Zircon (TEZ)}

\section{Eu/EuN*}

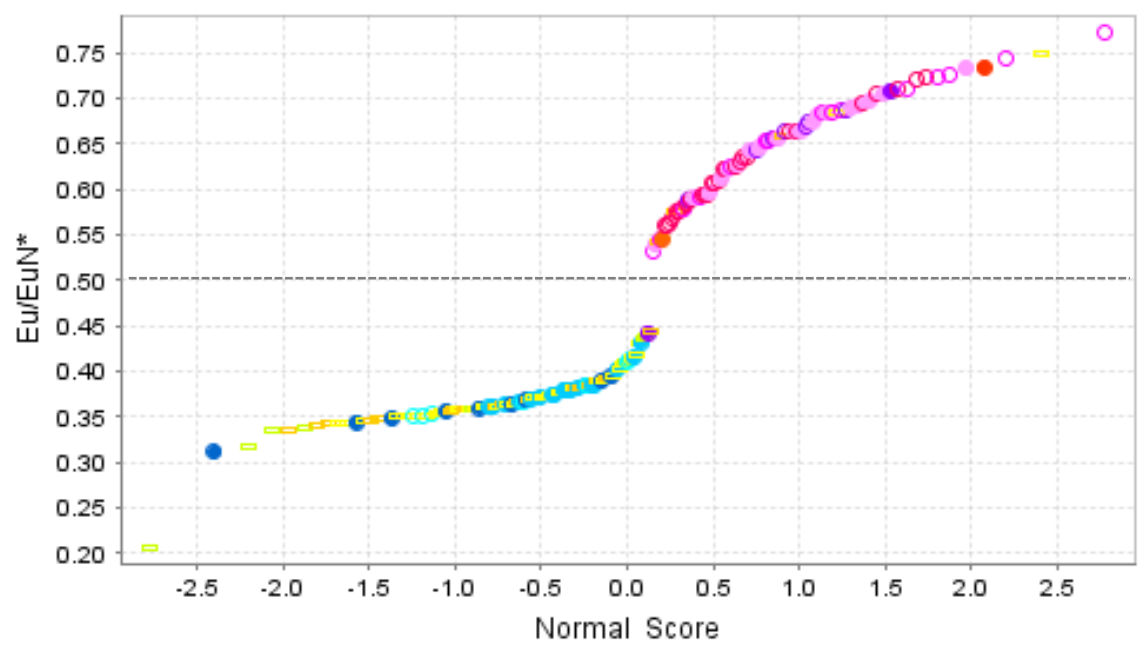

\section{Age Ma (Pb206/U238) : Eu/EuN*}

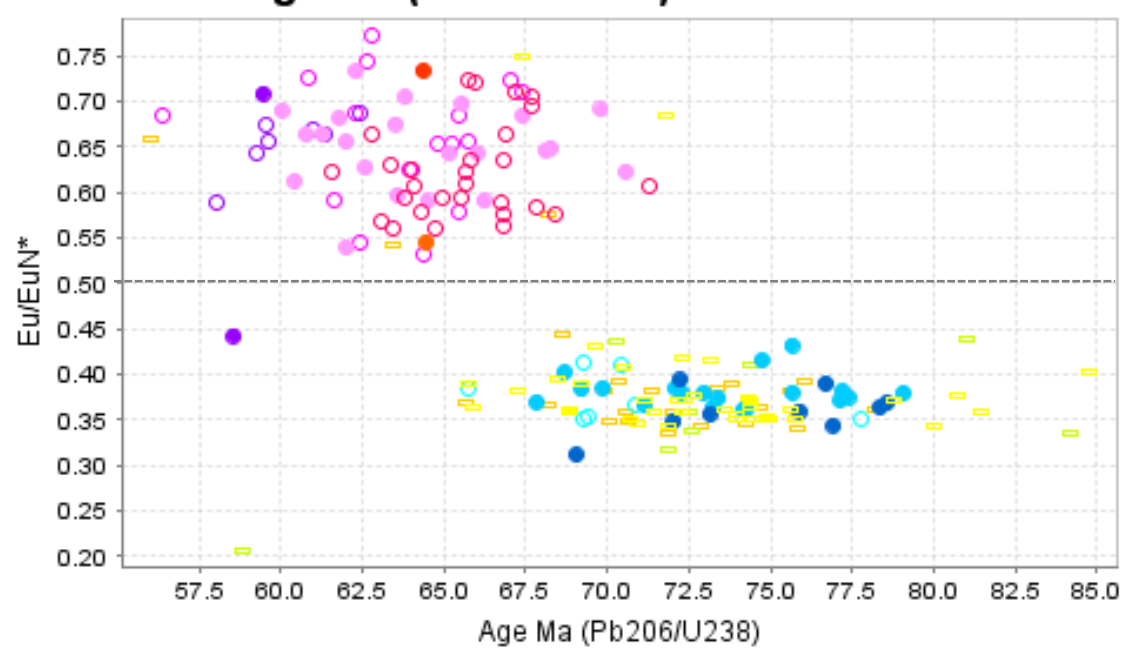




\section{Trace Eements in Zircon (TEZ) - Porphyry Fertility Indicators \\ Th/U : Eu/EuN* \\ $\mathrm{Th} / \mathrm{U}: \mathrm{Ce} / \mathrm{CeN}^{*}$}
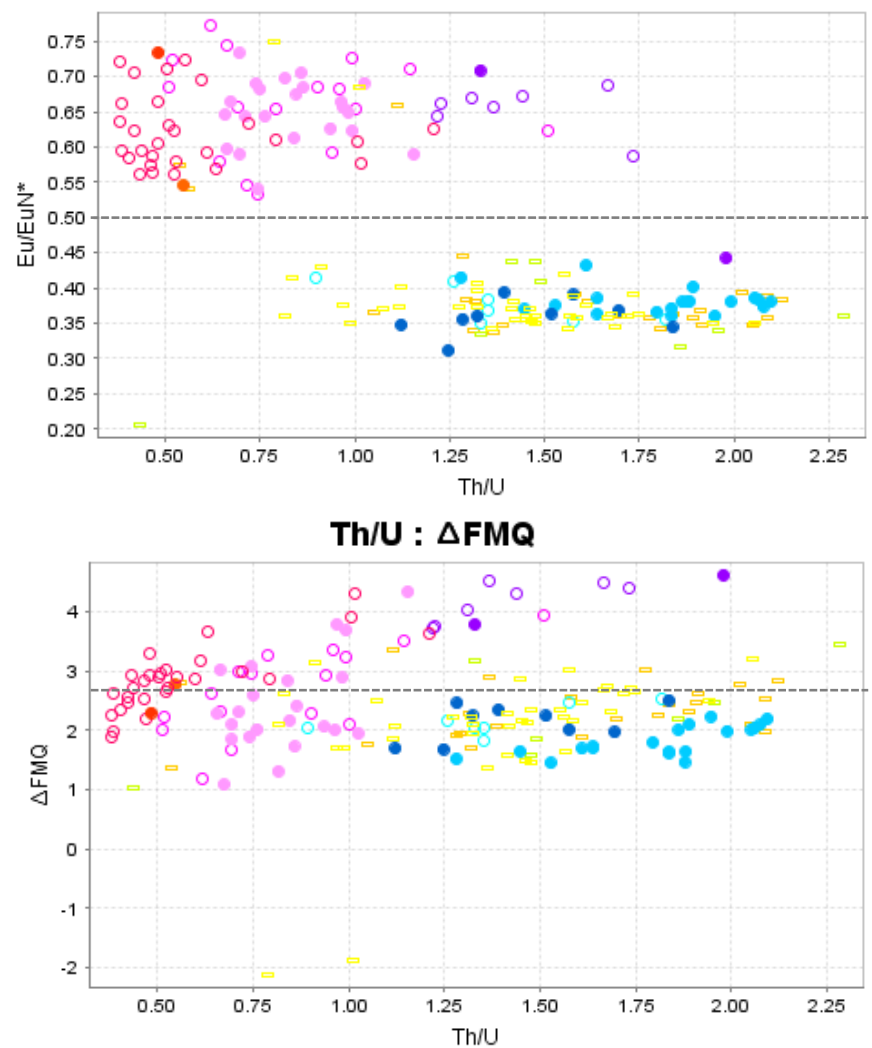

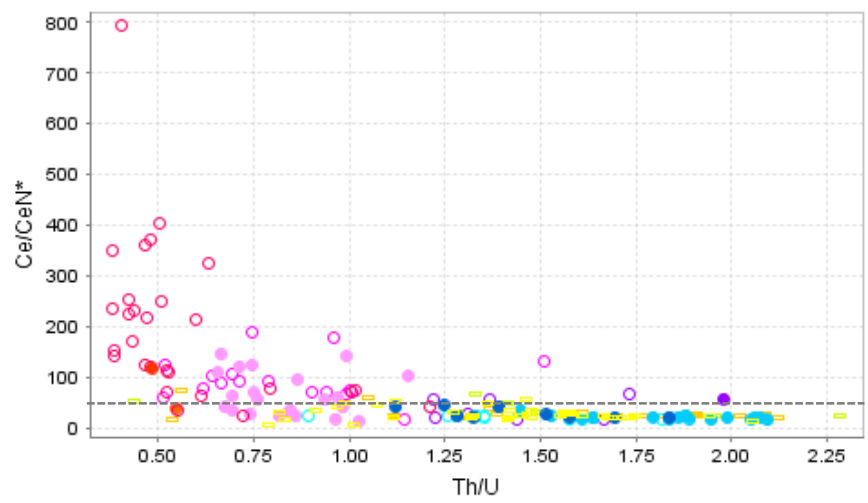

Th/U : (Ce/NdN)/Y

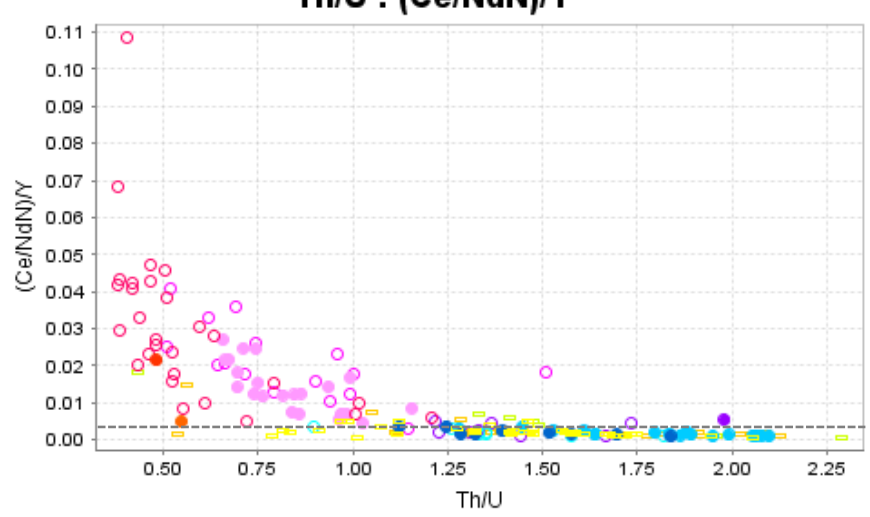

Christmas

Monzodiorite (Grauss)

Monzodiorite

Monzodiche, poinative autocryst

utocrysts

Ray

- Granite

Globe-Miami

Franodiorite

Fluvial-sand, Miami-Inspiration

Silver King

Fluvial-sand, Silver King

Quartz diorite (Grauss)

- Quartz diorite 


\section{Trace Elements in Zircon (TEZ) - Internal Zoning}
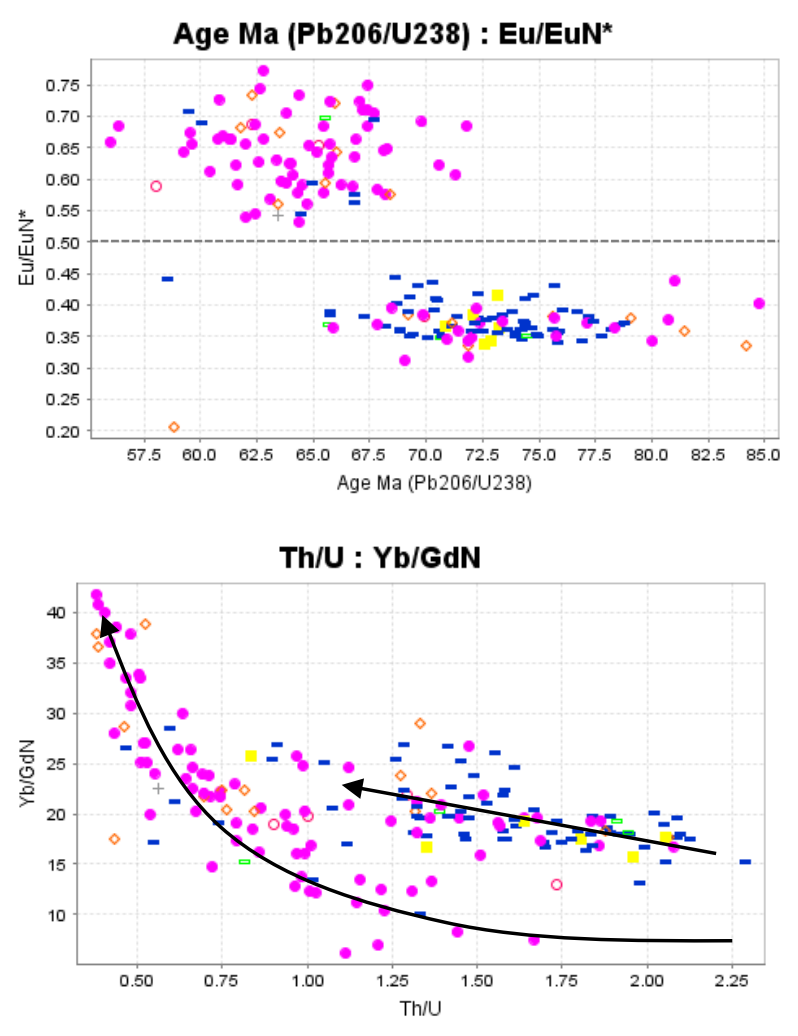

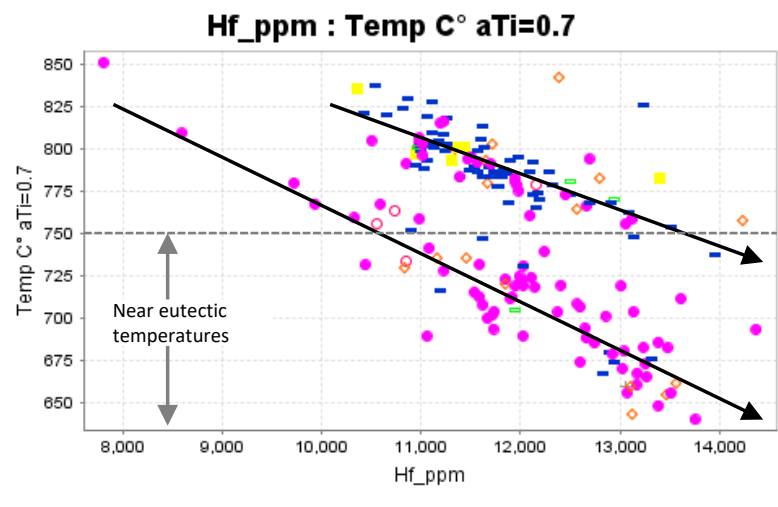

\section{Internal texture}

- oscillatory

0 oscillatory-irregular

$\ominus$ irregular

- tabular

- tabular-irregular tabular-oscillatory

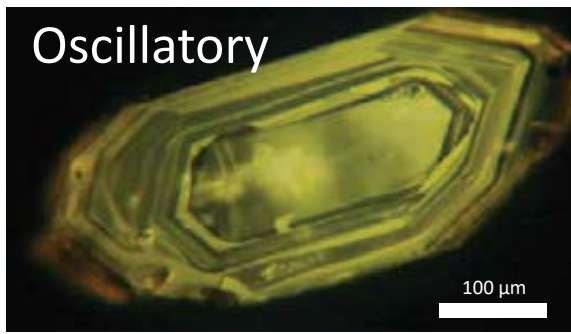

Tabular-oscillatory
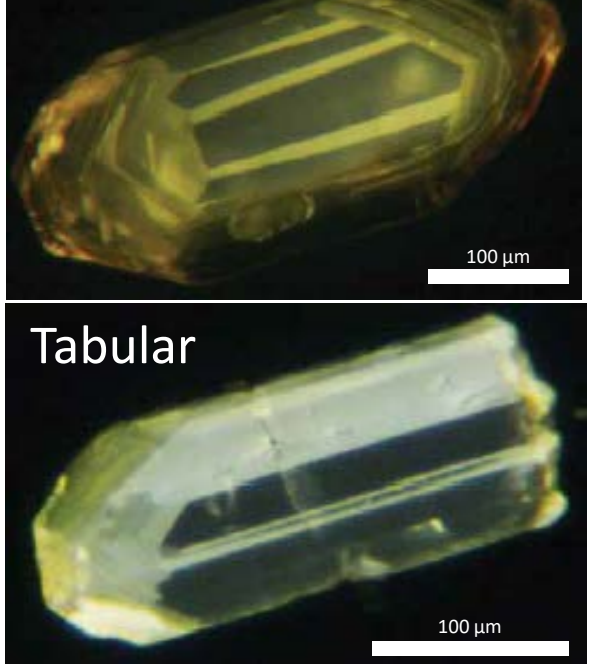


\section{Summary}

- Laramide plutonism in the district ranged from ca. 81 to $56 \mathrm{Ma}$.

- Magma chemistry continuously evolved throughout plutonism.

- Fractionation became amphibole dominated ca. 69 Ma.

- Younger intrusions were oxidized, hydrated and crystalized at lower temperatures.

- Late magmatism may have included mafic magma recharge.

- Zircon zoning textures can identify key porphyry-fertility and magmatic fractionation characteristics. 


\section{Thanks for listening}

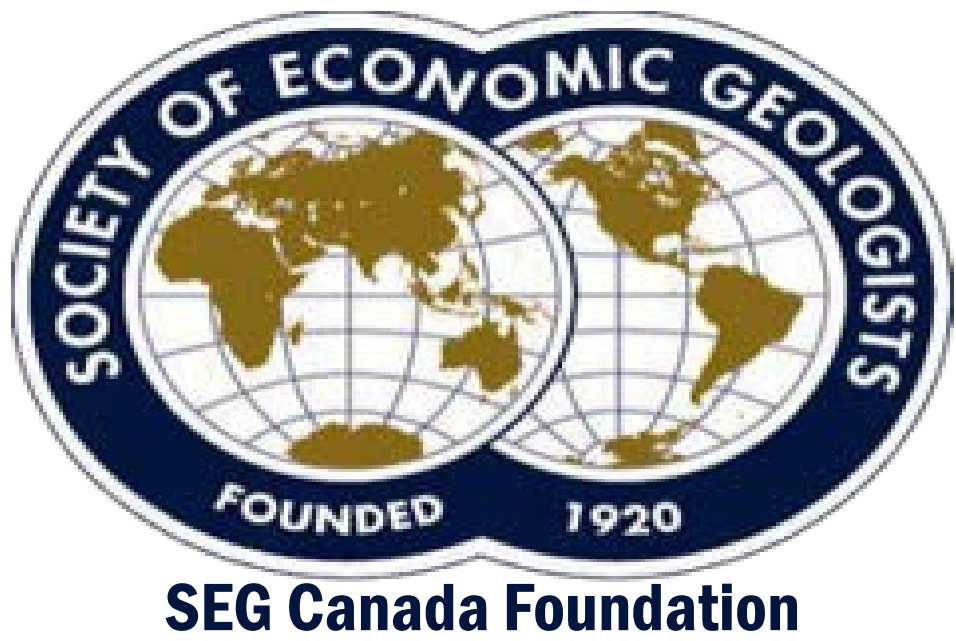

Corresponding author Taylor J. Ledoux tledoux@eoas.ubc.ca 